\title{
Defective Phagocytic Corpse Processing Results in Neurodegeneration and Can Be Rescued by TORC1 Activation
}

\author{
Jon Iker Etchegaray, ${ }^{1}$ Emma J. Elguero, ${ }^{1}$ Jennifer A. Tran, ${ }^{1}$ Vincent Sinatra, ${ }^{1}$ Mel B. Feany, ${ }^{2}$ and ${ }^{\odot}$ Kimberly McCall ${ }^{1}$ \\ ${ }^{1}$ Department of Biology, Boston University, Boston, Massachusetts 02215 and ${ }^{2}$ Department of Pathology, Brigham and Women's Hospital, Boston, \\ Massachusetts 02115
}

\begin{abstract}
The removal of apoptotic cell corpses is important for maintaining homeostasis. Previously, defects in apoptotic cell clearance have been linked to neurodegeneration. However, the mechanisms underlying this are still poorly understood. In this study, we report that the absence of the phagocytic receptor Draper in glia leads to a pronounced accumulation of apoptotic neurons in the brain of Drosophila melanogaster. These dead cells persist in the brain throughout the lifespan of the organism and are associated with age-dependent neurodegeneration. Our data indicate that corpses persist because of defective phagosome maturation, rather than recognition defects. TORC1 activation, or inhibition of Atg1, in glia is sufficient to rescue corpse accumulation as well as neurodegeneration. These results suggest that phagocytosis of apoptotic neurons by glia during development is essential for brain homeostasis in adult flies. Furthermore, it suggests that TORC1 regulates Draper-mediated phagosome maturation.
\end{abstract}

Key words: autophagy; cell death; Drosophila; glia; neurodegeneration; phagocytosis

\section{Significance Statement}

Previously, defects in dead cell clearance were linked to neurodegeneration, but the exact mechanisms are not well understood. In this study, we report that the absence of an engulfment receptor leads to a pronounced accumulation of dead neurons in the brain of the fruit fly Drosophila melanogaster. These dead cells persist in the brain throughout the lifespan of the organism and are associated with age-dependent neurodegeneration. Our data indicate that corpses persist because of defective degradation of cells rather than recognition of dead cells.

\section{Introduction}

Phagocytosis is a crucial component of development and homeostasis. Failure in the phagocytic process can result in a range of disease states (Poon et al., 2014). In the CNS, glia are responsible for executing the phagocytic response. These cells are important for the removal of dead cells, bacteria, and the refinement of circuits within the CNS (Chung et al., 2013; Sierra et al., 2013).

\footnotetext{
Received May 16, 2015; revised Jan. 18, 2016; accepted Jan. 22, 2016

Author contributions: J.I.E., M.B.F., and K.M. designed research; J.I.E., E.J.E., J.A.T., and V.S. performed research; M.B.F. contributed unpublished reagents/analytic tools; J.I.E., E.J.E., J.A.T., V.S., and K.M. analyzed data; J.I.E. and K.M. wrote the paper.

This work was supported by NIH Grants R01 GM094452 (K.M.) and F31 GM099425 (J.I.E.), BU Alzheimer's Disease Core Center NIH Grant P30 AG13846, Boston University Undergraduate Research Opportunities Program grants (J.A.T., V.S.), and NIH Grant R01 AG044113 to M.B.F. We thank the Bloomington Stock Center, TRiP at Harvard Medical School, the Kyoto Drosophila Genetic Resource Center, Estee Kurant, Eric Baehrecke, Marc Freeman, and Mary Logan for fly strains. We thank Todd Blute for assistance with electron microscopy and the Developmental Studies Hybridoma Bank for antibodies.

The authors declare no competing financial interests.

Correspondence should be addressed to Kimberly McCall at the above address. E-mail: kmccall@bu.edu. DOI:10.1523/JNEUROSCI.1912-15.2016

Copyright $\odot 2016$ the authors $\quad 0270-6474 / 16 / 363170-14 \$ 15.00 / 0$
}

Phagocytic glia have been observed in nearly all neurological diseases (Fu et al., 2014). Genome wide association studies have found a correlation between various alleles of genes involved in glia engulfment and risk of developing neurodegenerative disorders (Kauwe et al., 2014). Mechanisms underlying phagocytosis are not well understood, and how defects in clearance of apoptotic cells contribute to neurodegeneration remain understudied.

Phagocytosis of dead cells can be subdivided into three steps (Elliott and Ravichandran, 2010). The first step involves recognition of the dead cell by the phagocyte via exposure of "eat me" signals on the membrane of the dead cell. Phagocytes employ a complementary array of recognition receptors that bind to the "eat me" signals. Binding of a recognition receptor to an "eat me" signal triggers intracellular events that lead to cytoskeletal rearrangements that result in internalization of the dead cell into a vesicle, known as a phagosome, within the phagocyte. Phagosomes undergo successive fusion events to endosomes and lysosomes, culminating in degradation of the apoptotic cell within the phagocyte (known as corpse processing or phagosome maturation). 
How a cell is able to distinguish vesicles containing apoptotic cells as opposed to other endocytosed material remains poorly understood. Previous findings have demonstrated that some apoptotic recognition receptors are important for internalization of corpses and phagosome maturation (Lu and Zhou, 2012). This dual role may allow cells to distinguish apoptotic versus necrotic cells/pathogens and execute the appropriate response by coupling recognition to a specific set of processing signals (Blander and Medzhitov, 2006). Given this dual role, disrupting these receptors may have a disproportional impact on phagocytosis and homeostasis. In fact, one of the strongest risk factors for Alzheimer's disease is a variant of the gene TREM2 (Guerreiro et al., 2013; Jonsson et al., 2013). TREM2 was shown previously to be a dual-role phagocytic recognition receptor (Cantoni et al., 2015). Better understanding of signaling mechanisms employed by this class of receptors may be crucial to elucidating how defective phagocytosis by glia affects brain homeostasis.

Work in Caenorhabditis elegans has identified two partially redundant phagocytic pathways, known as the CED-1/CED-6/ CED-7 and CED-2/CED-5/CED-12 pathways, which show a high degree of conservation from invertebrates to mammals (Hochreiter-Hufford and Ravichandran, 2013). In these pathways, it has been shown that CED-1 and its orthologs in mammals, MEGF10 and Jedi (Scheib et al., 2012), act as recognition receptors. CED-1 has also been shown to be important in phagosome maturation. Specifically, CED-1 leads to the recruitment of dynamin, a large GTPase involved in processing nascent phagosomes (Yu et al., 2008). Whether MEGF10 or Jedi is important for corpse processing is not known.

Drosophila melanogaster has been a powerful model for understanding glia-dependent phagocytosis and neurodegenerative diseases (Shulman et al., 2003; Fullard et al., 2009). The CED-1 ortholog Draper (Drpr) is an important recognition receptor for glia-dependent phagocytosis (Freeman et al., 2003). Drpr participates in corpse processing as well as recognition (Kurant et al., 2008). However, signaling mechanisms important for Drprdependent corpse processing are unknown. The dual function of Drpr in phagocytosis, as well as its high conservation across species, led us to investigate whether defects in Drpr-dependent glia phagocytosis cause disruptions in brain homeostasis. We found that the absence of Drpr in glia led to a massive accumulation of neuronal corpses in adult brains and neurodegeneration. Our findings indicate that these phenotypes arise from ineffective corpse processing rather than defects in recognition. These phenotypes were rescued by inactivating autophagy through TORC1 activation, pointing to a novel role for TORC1 in regulating phagosome maturation.

\section{Materials and Methods}

Fly strains and manipulations. The following strains were obtained from the Bloomington Stock Center: repo-GAL4, elav-GAL4 (c155), tubPGAL80 $^{\text {ts }}$ (7016), UAS-IVS-myr::GFP, UAS-His2A-RFP, UAS-GFP RNAi, UAS-Rab7::GFP, UAS-LAMP::GFP, UAS-Atg1 RNAi, UAS-Rheb, UASAtg7 RNAi, UAS-Atg8a RNAi, UAS-Atg12 RNAi, UAS-Atg16 RNAi, and UAS-Ref2P RNAi. $d r p r^{\Delta 5}$ (Freeman et al., 2003) was provided by Estee Kurant (Technion, Haifa, Israel). UAS-drpr, UAS-drpr RNAi (MacDonald et al., 2006), and UAS-drpr-I RNAi (McPhee et al., 2010) were provided by Mary Logan (Oregon Health and Science University, Portland, OR) and Marc Freeman (University of Massachusetts Medical School, Worcester, MA). Atg8a-GFP was provided by Eric Baehrecke (University of Massachusetts Medical School). The cortex glial subtype GAL4 driver (NP2222) was obtained from the Drosophila Genomics Resource Center. Fly crosses were performed at $25^{\circ} \mathrm{C}$, except for crosses in Figure $4 E-H$. Briefly, these crosses were kept at $18^{\circ} \mathrm{C}$ until respective times in develop- ment and then transferred to $29^{\circ} \mathrm{C}$ where the progeny eclosed. Males were used in Figures 1 and 2, and both sexes were used for the other figures.

Antibody staining and microscopy. Flies were dissected in 4\% paraformaldehyde diluted in $1 \times$ PBS buffer plus $0.1 \%$ Triton X-100 (PBT) and fixed for $50 \mathrm{~min}$. Brains were washed three times in PBT, $15 \mathrm{~min}$ each, while rotating, and then samples were placed in VectaShield with DAPI (Vector Labs) overnight at $4^{\circ} \mathrm{C}$ and subsequently mounted on slides. For antibody staining, after three washes in PBT, brains were blocked for $1 \mathrm{~h}$ in PBT plus $0.5 \%$ BSA plus $5 \%$ normal goat serum (PBANG) and then placed in antibody diluted in PBANG for $4 \mathrm{~d}$. Brains were washed three times in PBT plus $0.5 \%$ BSA and then placed in secondary antibody diluted in PBANG for $4 \mathrm{~h}$. Brains were then washed again three times in PBT plus $0.5 \%$ BSA. Samples were placed in VectaShield with DAPI (Vector Labs) overnight at $4^{\circ} \mathrm{C}$ and subsequently mounted. The following primary antibodies were used: rabbit anti-cleaved Drosophila Dcp-1 (Cell Signaling Technology) used at a dilution of 1:100, mouse antiDraper (Developmental Studies Hybridoma Bank) used at 1:10, mouse anti-Discs Large (Developmental Studies Hybridoma Bank) used at 1:500, and rabbit anti-GFP (Torey Pines) used at 1:500. Secondary antibodies were Cy3 anti-rabbit IgG used at 1:200, Alexa 488 anti-mouse IgG used at 1:400, and Alexa 488 anti-rabbit IgG used at 1:400; Jackson ImmunoResearch) . Brains were imaged on an Olympus FV10i confocal microscope. All $z$-projection images consisted of $301 \mu \mathrm{m}$ stacks starting from the surface of the brain from the first point where the antennal lobe becomes visible. Images were processed in Fiji and Adobe Photoshop.

TUNEL staining. The DeadEnd Fluorometric TUNEL System (Promega) was used. Briefly, after fixing and washing as for antibody staining, tissue was incubated for $10 \mathrm{~min}$ in $20 \mu \mathrm{l}$ of equilibration buffer from the kit while rotating. The equilibration buffer was removed and recombinant terminal deoxynucleotidyl transferase (rTdT) incubation buffer was added ( $45 \mu \mathrm{l}$ equilibration buffer, $5 \mu \mathrm{l}$ nucleotide mix, $1 \mu \mathrm{l}$ rTdT enzyme). Samples were then incubated for $3 \mathrm{~h}$ in a $37^{\circ} \mathrm{C}$ water bath. The reaction was stopped by adding $300 \mu \mathrm{l}$ of $2 \times$ SSC solution for $1 \mathrm{~min}$ while rotating. The solution was removed and replaced with another 300 $\mu \mathrm{l}$ of $2 \times$ SSC, rotating for $15 \mathrm{~min}$. Tissue was washed three times in PBT while rotating for a total of $30 \mathrm{~min}$. Samples were then placed in VectaShield with DAPI. To quantify the number of TUNEL-positive cells, we used the 3D object counter plugin in Fiji. Unpaired $t$ tests were used for all statistical analyses.

Sectioning. For histology, flies were processed for paraffin embedding and sectioned at a thickness of $4 \mu \mathrm{m}$. Sections from the entire brain were placed on a single glass slide and stained with hematoxylin and eosin. For quantifications, vacuoles $4 \mu \mathrm{m}$ or greater in size were counted. Researchers were blinded during quantifications. Unpaired $t$ tests were used for all statistical analyses.

Electron microscopy. Fly brains were dissected in 4\% paraformaldehyde in PBS and fixed for $2 \mathrm{~h}$ on ice in $2 \%$ glutaraldehyde. The brains were then washed three times for $10 \mathrm{~min}$ in $0.1 \mathrm{M}$ sodium cacodylate buffer. Brains were postfixed in $1 \%$ osmium tetroxide for $2 \mathrm{~h}$ at RT. Brains were then washed four times for $5 \mathrm{~min}$ each in $\mathrm{dH}_{2} \mathrm{O}$. Brains were incubated in $1 \%$ uranyl acetate for $30 \mathrm{~min}$ and then washed three times for $10 \mathrm{~min}$ each in $\mathrm{dH}_{2} \mathrm{O}$. Brains were then dehydrated through a series of ethanol washes and washed for $1 \mathrm{~h}$ in 100\% propylene oxide before being washed overnight in 1:1 propylene oxide/resin solution. Brains were then transferred to $100 \%$ Epon 812 resin for $2 \mathrm{~h}$ and then embedded in flat molds with fresh resin and allowed to polymerize at $60^{\circ} \mathrm{C}$ for at least $48 \mathrm{~h}$. Ninetyfive nanometer sections were cut and examined with a Tecnai G2 Spirit BioTWIN transmission electron microscope at an accelerating voltage of $80 \mathrm{kV}$.

\section{Results}

\section{Apoptotic corpses persist in $d r p r^{-/-}$brains}

To determine the impact of defective phagocytosis on brain homeostasis, we examined the brains of $d r p r^{-1-}$ flies for persistent cellular corpses. Specifically, we looked for the presence of pyknotic nuclei, highly condensed chromatin suggestive of dead cells (Savill et al., 2002). We dissected brains from 1-d-old 

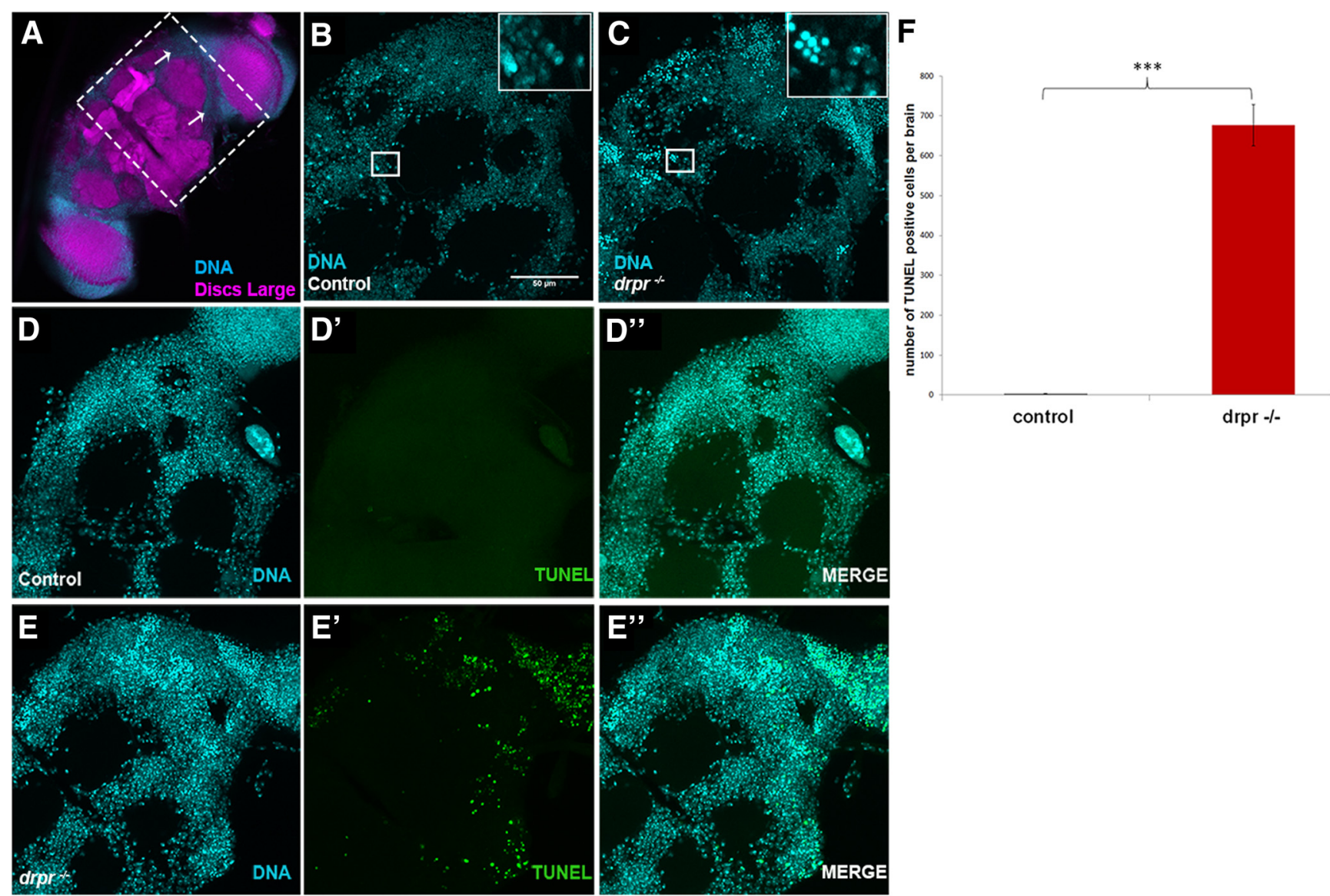

Figure 1. Draper is required for corpse clearance in the adult brain. $A$, Brain stained with DAPI (blue) to label nuclei (arrows) and anti-Discs large (magenta) to label neuropil. The white box represents the area of the brain that is shown in subsequent images. All images are $z$ projections unless noted otherwise. $B, C$, DAPI staining shows normal nuclear morphology in control $w^{1718}$ ( $\boldsymbol{B}$ )

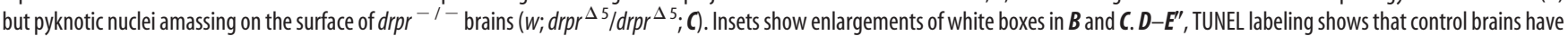
few TUNEL-positive cells $\left(\boldsymbol{D}^{\prime}, \boldsymbol{D}^{\prime \prime}\right)$, whereas drpr null brains contain a large number of positive cells $\left(\boldsymbol{E}, \boldsymbol{E}^{\prime \prime}\right) . \boldsymbol{F}$, Quantification of TUNEL-positive cells in the central brain of 1-d-old control and $d r p r$ null brains $(n \geq 6)$. ${ }^{* *} p \leq 0.001$. Data presented are mean \pm SEM.
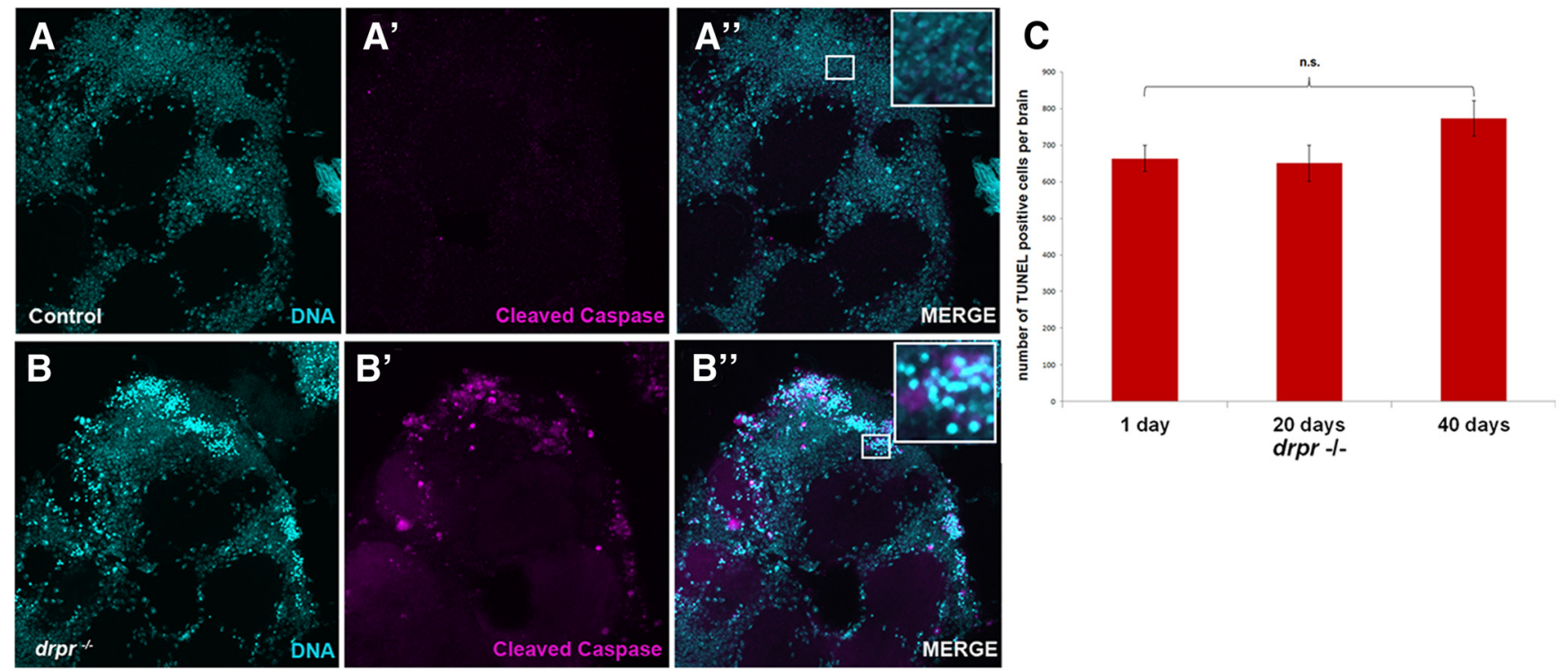

Figure 2. The absence of draper leads to persistence of apoptotic cells in the brain over the lifespan of the fly. $A-B^{\prime \prime}, w^{1118}$ brains are mostly negative for anti-cleaved Dcp-1 caspase staining (magenta; $\boldsymbol{A}, \boldsymbol{A}^{\prime}$ ), whereas $\mathrm{drpr}^{-1-}$ brains are positive for anti-cleaved Dcp-1 $\left(\boldsymbol{B}^{\prime}\right)$ that overlaps with pyknotic nuclei $\left(\boldsymbol{B}^{\prime \prime}\right)$. Insets show enlargements of boxes in $\boldsymbol{A}^{\prime \prime}$ and $\boldsymbol{B}^{\prime \prime}$. $\boldsymbol{C}$, Quantification of TUNEL-positive cells in the brain of 1-, 20-, and 40-d-old drpr null brains ( $n \geq 8$ ). Error bars indicate SEM. 
$d r \mathrm{pr}^{-/-}$flies, stained them with DAPI, and surveyed the surface of the brain, where most cell bodies are located (Ito et al., 2014; Fig. 1A). The nuclei of control $\left(w^{1118}\right)$ brains appeared normal, with diffuse uncondensed DNA and a single bright condensed speckle corresponding to heterochromatin (Frost et al., 2014; Fig. $1 B)$. As with control brains, the majority of nuclei in $d r p r^{-1-}$ brains had normal chromatin morphology (Fig. 1C); however, a large number of the nuclei had a highly condensed appearance that was uniform throughout the nucleus, indicating pyknosis (Fig. 1C). These pyknotic nuclei were found throughout the surface of the central brain as well as the surface of the optic lobes (Fig. 1C). To confirm that the large number of pyknotic nuclei seen in $d r \mathrm{pr}^{-1-}$ brains were dead cells, we performed TUNEL labeling, which detects fragmented DNA, as well as antibody staining for cleaved Dcp-1, an effector caspase (Song et al., 1997). Control flies displayed one to two TUNEL-positive cells per brain at $1 \mathrm{~d}$ of age (Fig. $\left.1 D^{\prime}, F\right) \cdot d r p r^{-/-}$brains displayed a much higher number of TUNEL-positive nuclei, with an average of 663 dead cells per brain (Fig. $1 E^{\prime}, F$ ). Furthermore, TUNEL labeling in $\mathrm{drpr}^{-1-}$ brains highly colocalized with pyknotic nuclei as detected with DAPI staining (Fig. $\left.1 E^{\prime \prime}\right) . d r p r^{-1-}$ brains were also highly positive for cleaved Dcp-1 compared to control (Fig. $\left.2 A^{\prime}, B^{\prime}\right)$. As with TUNEL staining, the brain regions with high levels of cleaved Dcp-1 corresponded to the regions of pyknotic nuclei (Fig. $2 B^{\prime \prime}$ ). These results indicate that the pyknotic nuclei correspond to apoptotic corpses. These experiments were performed on 1-d-old flies, so we were interested in determining the fate of these corpses as flies aged. We compared TUNEL labeling on flies at 1, 20, and $40 \mathrm{~d}$ of age. To our surprise, the number of TUNEL-positive corpses did not change with time (Fig. 2C). This suggests that corpses remain in the brain throughout the lifespan of the organism.

The above analyses were conducted on $d r p r$ null flies. The defect in corpse clearance could be due to an absence of $d r p r$ in glia, neurons, or macrophages outside of the brain. To determine where $d r p r$ was required to prevent the persistence of apoptotic cells, we knocked down $d r p r$ in specific cell types with the GAL4UAS system (Brand and Perrimon, 1993). We found that pyknotic nuclei formed in the brains of flies expressing $d r p r$ RNAi in glia using repo-GAL4 (Fig. $3 A, B$ ) but other tissue-specific knockdowns had no effect. As reported previously (MacDonald et al., 2006), we confirmed that $d r p r$ RNAi efficiently suppressed the expression of Draper when driven in glial cells (Fig. 3C,D). The large number of pyknotic nuclei that formed with repo-GAL4 driving $d r p r$ RNAi were also TUNEL positive, indicating that they were apoptotic corpses (Fig. 3E-G). To demonstrate specificity of the RNAi, we were able to rescue the phenotype by overexpression of $d r p r$ (Fig. 3G).

The $d r p r$ gene encodes three different isoforms with distinct functions. The first isoform positively regulates phagocytosis while the second negatively regulates engulfment (Logan et al., 2012). The function of the third isoform is unknown (Logan et al., 2012). The RNAi line we used above knocks down all three isoforms. To narrow down which isoform is responsible for the corpse phenotype, we knocked down $d r p r$ I and found that it was sufficient to cause the phenotype (Fig. $3 H$ ). As with mammals, Drosophila contain different glia subtypes in the brain with different function. These include astrocyte, ensheathing, cortex, perineurial, and subperineurial glia (Awasaki et al., 2008). To determine which glia subtype acted as phagocytes to remove apoptotic corpses, we knocked down $d r p r$ in the individual glia subtypes with different glia-specific GAL4 drivers. We found that expressing $d r p r$
RNAi in cortex glia led to persistent pyknotic nuclei (Fig. $3 I, J)$. Knocking down $d r p r$ in the other glial subtypes had no effect. Together, these data indicate that $\operatorname{drpr}$ (isoform I) is required in cortex glia to prevent the accumulation of apoptotic corpses in the brain. Furthermore, once these corpses form, they likely remain in the brain for the lifespan of the organism.

\section{The apoptotic corpses are neurons that died during late larval and early pupal development}

Our results indicate that pyknotic nuclei correspond to apoptotic cells that have not been cleared. However, the cellular identity of these corpses remained elusive, as they did not label with antibodies against neuronal or glial proteins, which are typically lost early during cell death. The Drosophila brain is predominantly composed of neurons, with glia constituting approximately only $10 \%$ of the cells (Edwards and Meinertzhagen, 2010). The high number of corpses led us to believe that they were likely neurons. To determine whether the corpses were neurons, we marked neuronal membranes with myristoylated GFP using elav-GAL4 in a $d r \mathrm{pr}^{-1-}$ background. In control brains, myristoylated GFP surrounded neurons in a membrane-like fashion (Fig. $4 A-D)$. In the $d r \mathrm{pr}^{-/-}$background, GFP collapsed around corpses (Fig. 4E-H), suggesting that they were neuronal in origin. To further confirm that the corpses were neurons, we expressed a fluorescently tagged version of Histone 2A, His2A-RFP, in neurons in a $d r p r^{-1-}$ background. We found that corpses were positive for His 2A-RFP (Fig. 5A-B" ), indicating that they were indeed apoptotic neurons. As a control, we expressed His2A-RFP in glia using the repo-GAL4 driver in $d r p r^{-1-}$ brains and found that corpses largely did not colocalize with His2A-RFP (Fig. 5C-D"). These findings indicate that the majority of corpses are dead neurons.

Drosophila has five discrete stages of development: embryo; first, second, and third instar larva; and pupa. The nervous system has been shown to undergo programmed cell death at four different time points during these stages of development: mid to late embryogenesis, third instar larval phase, metamorphosis during pupal stage, and in the newly eclosed adult (Gilbert, 2012). To determine the specific time point in development that these corpses formed, we used a temperature-sensitive form of GAL80 (a repressor of the UAS-GAL4 system; McGuire et al., 2003) to induce $d r p r$ RNAi expression in glia at different time points during development. Specifically, larvae were shifted at different developmental time points from $18^{\circ} \mathrm{C}$, a temperature at which GAL80 actively represses expression of the UAS-driven transgene, to $29^{\circ} \mathrm{C}$, a temperature at which GAL 80 degrades and therefore the UAS-driven transgene can become active. We observed that pyknotic nuclei were present in the central brain of adult flies when they had been shifted to $29^{\circ} \mathrm{C}$ at the second instar phase of larval development (Fig. $4 I, M$ ). Corpses were mostly absent when flies were shifted to $29^{\circ} \mathrm{C}$ at the beginning of the third instar phase of larval development (Fig. 4J,M). Corpses in the optic lobe, however, were still present at this time (Fig. $4 K$ ). Conversely, when flies were shifted to $29^{\circ} \mathrm{C}$ toward the end of larval development, corpses were absent from the optic lobe (Fig. $4 L$ ). These data suggest that the corpses that are observed in the central brain and optic lobe of adult $d r \mathrm{pr}^{-/-}$flies form during the beginning and late portions of the third instar larval phase, respectively. Surprisingly, our findings also indicate that dying neurons arising later in development are efficiently cleared without Drpr. 

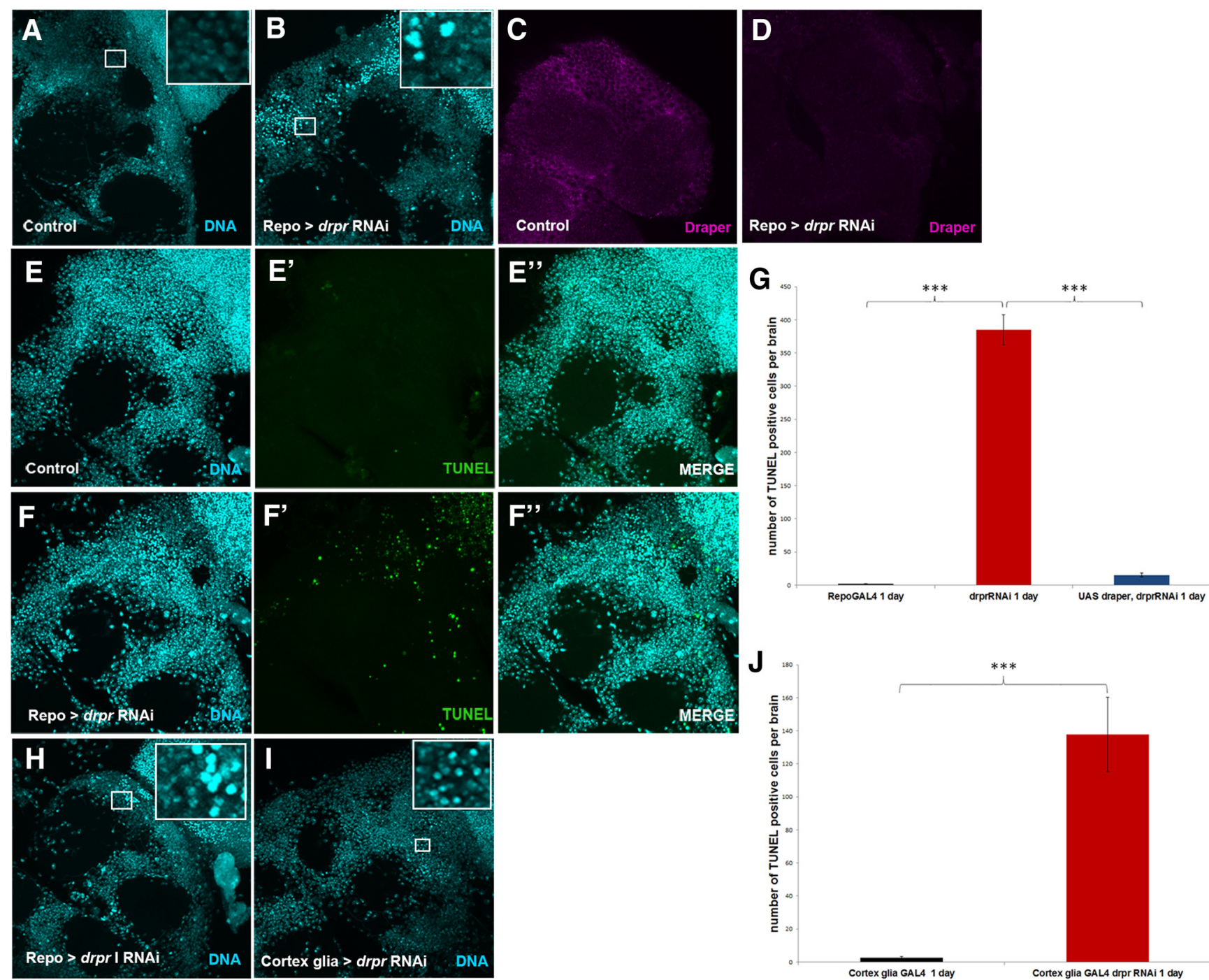

Figure 3. Knockdown of draper in glia leads to corpse accumulation. $A, B, D A P I$ staining on control (repo-GAL4) brain shows typical DNA morphology ( $\boldsymbol{A})$, whereas DAPI staining of draper RNAi driven by repo-GAL4 in glia (UAS-drpr-RNAi/+; repoGAL $4 /+; \boldsymbol{B})$ shows pyknotic nuclei on the surface of the brain. $\boldsymbol{C}, \boldsymbol{D}$, Control brains are immunoreactive to Draper antibody $(\boldsymbol{C})$, whereas brains where drpr RNAi is expressed in glia are not (D). Images in $\boldsymbol{C}$ and $\boldsymbol{D}$ are single slices. $\boldsymbol{E}-\boldsymbol{F}^{\prime \prime}$, Control brains are mostly TUNEL negative $\left(\boldsymbol{E}^{\prime}\right)$, whereas drpr RNAi driven in glia results in a large number of TUNEL-positive cells $\left(\boldsymbol{F}^{\prime}\right)$. G, Quantification of TUNEL-positive cells in the central brain of 1-d-old control, drpr RNAi, and rescued brains $(n \geq 6)$. ${ }^{* * *} p \leq 0.001$. $\boldsymbol{H}, \mathrm{DAPI}$ staining of a brain in which drpr I RNAi is driven by repo-GAL4 (UAS-draper-I-RNAi/repo-GAL4) shows pyknotic nuclei on the surface. I, DAPI staining of draper RNAi being driven by a cortex glia-specific promoter (UAS-draperRNAi/+;NP2222-GAL4/+) shows pyknotic nuclei accumulating on the surface of the brain. J, Quantification of TUNEL-positive cells in the central brain of 1-d-old draper RNAi driven in cortex glia and control brains $(n \geq 6) .{ }^{* *} p \leq 0.001$. Insets show enlargements of white boxes in $\boldsymbol{A}, \boldsymbol{B}, \boldsymbol{H}$, and $\boldsymbol{I}$. Error bars indicate SEM.

\section{Knockdown of $d r p r$ in glia leads to age-dependent neurodegeneration and glial death}

We next wanted to determine whether the persistence of neuronal apoptotic corpses in $d r p r$ mutant flies would disrupt brain homeostasis, potentially leading to neurodegeneration. Thus, we sectioned $\mathrm{drpr}^{-/-}$brains at 1 and $40 \mathrm{~d}$ of age and examined them for the presence of vacuoles, a hallmark of neurodegeneration in Drosophila (Celotto and Palladino, 2005; Hegde et al., 2014). We found that $\mathrm{rrpr}^{-/-}$brains exhibited high levels of vacuolization at $40 \mathrm{~d}$ compared to control but there was no difference between 1-d-old brains. We then repeated this experiment in flies that had drpr specifically knocked down in glia. At $1 \mathrm{~d}$ of age, brains expressing $\operatorname{drpr}$ RNAi or GFP RNAi control in glial cells did not show high levels of vacuolization (Fig. $6 A, B, E$ ). However, at $40 \mathrm{~d}$ of age, brains expressing $\operatorname{drpr}$ RNAi in glia, but not GFP RNAi, showed significant levels of vacuolization (Fig. $6 C-E$ ). Consistent with our results, neurodegeneration in $\mathrm{drpr}^{-/-}$brains was reported previously (Draper et al., 2014). These experiments show that $d r p r$ is required in glia for brain homeostasis and the prevention of age-dependent neurodegeneration.

Our previous work has shown that knocking down $d r p r$ in another model of engulfment, the Drosophila ovary, leads to premature death of the phagocytes (Etchegaray et al., 2012). In mammals, mutations in other phagocytosis genes have also been shown to cause death of the phagocyte (Yvan-Charvet et al., 2010). We were interested to see if knockdown of $d r p r$ in glia also led to glial death, which could be a cause of the neurodegeneration. We used His2A-RFP driven by repo-GAL4 to mark glia and stained for TUNEL to visualize cell death. Control brains of flies expressing His2A-RFP in glia showed few to no TUNEL-positive glia (Fig. $7 A-A^{\prime \prime \prime}, C$ ). In contrast, brains of flies that expressed His2A-RFP and $d r p r$ RNAi showed some overlap between TUNEL and RFP, indicating glia death (Fig. $\left.7 B-B^{\prime \prime \prime}, C\right)$. Furthermore, it appeared that glia that were TUNEL positive were actively dying, as opposed to the majority of corpses, which were neurons that died previously and persisted in the brain. This 

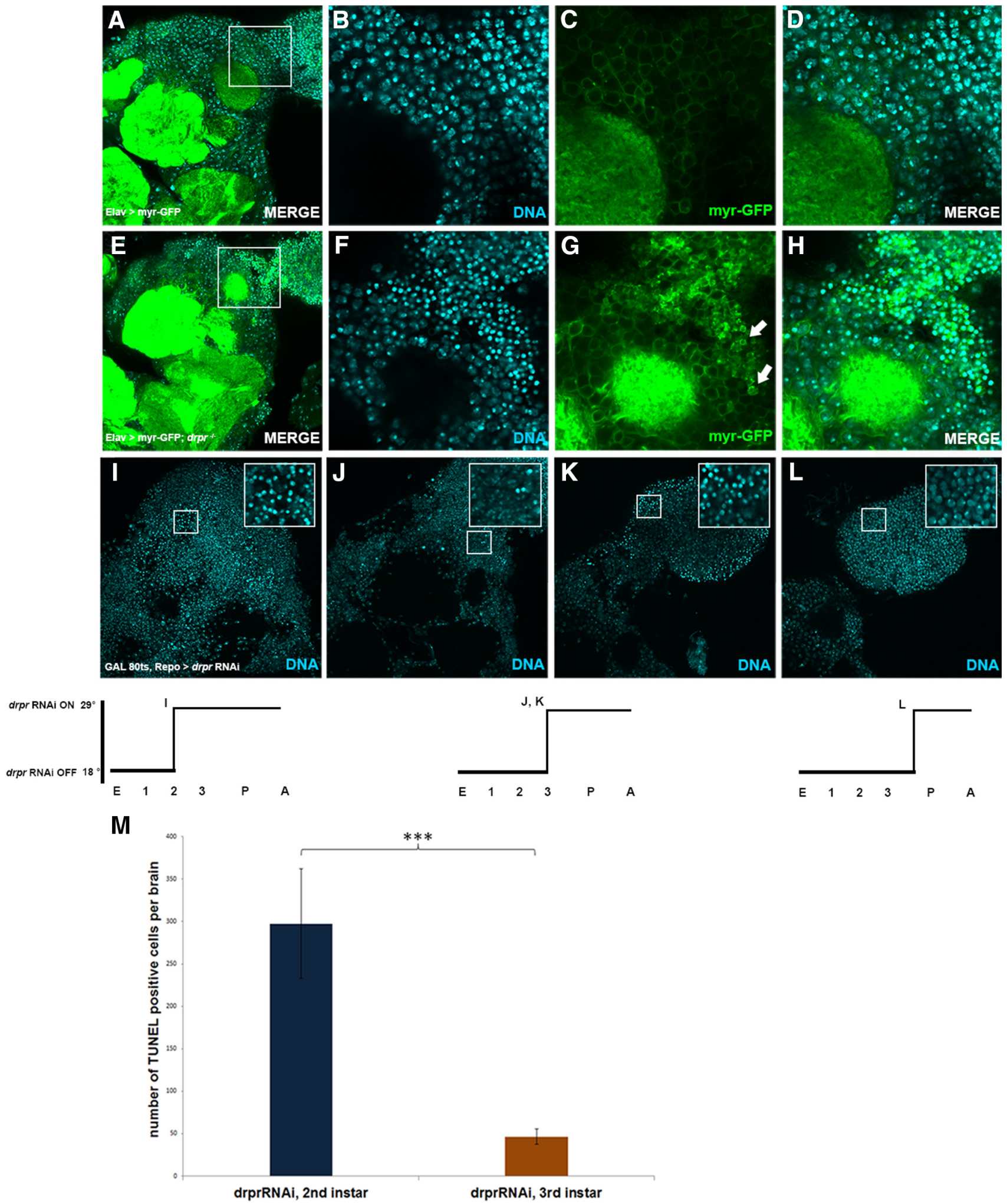

Figure 4. Neuronal corpses form during development in draper deficient brains. A, DAPI stain on brains that express myristoylated-GFP under elav-GAL4 (elav-GAL4/+; UAS-IVS-myristoylated::GFP/+) shows GFP surrounding neuronal nuclei. $\boldsymbol{B}-\boldsymbol{D}$, Zoom of the white box in $\boldsymbol{A}$ shows myristoylated-GFP ( $\boldsymbol{C}$ ) surrounding neuronal nuclei (B) in $\boldsymbol{D}$. $\boldsymbol{E}$, DAPI stain on drpr null brains that also express myristoylated-GFP under elav-GAL4 (elav-GAL4/+; UAS-IVS-myristoylated::GFP/+; $\mathrm{drpr}^{\Delta 5} / \mathrm{drpr}^{\Delta 5}$ ) shows GFP collapsed around pyknotic nuclei. $\boldsymbol{F}$-H, Zoom of the white box in $\boldsymbol{E}$ shows myristoylated-GFP $(\boldsymbol{G})$ surrounding pyknotic nuclei (G, arrows) in $\boldsymbol{H}$. I, J, Corpses persist in the adult central brain of tub-GAL80 $/+$; UAS-draper-RNAi/+; repo-GAL4/+ flies when second instar larvae are shifted to $29^{\circ} \mathrm{C}(\boldsymbol{I})$ and largely disappear when flies are shifted to $29^{\circ} \mathrm{C}$ during the beginning of the third instar larval stage $(\boldsymbol{J})$. $K, \boldsymbol{L}$, Corpses persist in the adult optic lobes of tub-GAL8OS/ $/$;UAS-draper-RNAi/+; repo-GAL4/+ flies when larvae are shifted to $29^{\circ} \mathrm{C}$ during the beginning of the third instar larval stage $(\boldsymbol{K})$ and largely disappear if flies are shifted to $29^{\circ} \mathrm{C}$ during at the end of the third instar larval stage $(\boldsymbol{L})$. Below the images are schematics for the temperature shift protocol used for $\boldsymbol{I}-\boldsymbol{L}$. Briefly, flies were raised at $18^{\circ} \mathrm{C}$, shifted to $29^{\circ} \mathrm{C}$ at different time points during development, and raised at $29^{\circ} \mathrm{C}$ until eclosion. E, Embryo; 1 , first instar larva; 2 , second instar larva; 3 , third instar larva; $\mathrm{P}$, pupa; $\mathrm{A}$, adult. Images from $\boldsymbol{A - H}$ are all single-slice images. M, Quantification of TUNEL-positive cells in the central brain of tub-GAL80 $/++, U A S-d r p r-R N A i /+;$ repo-GAL4/+ flies when second instar larvae are shifted to $29^{\circ} \mathrm{C}$ and when third instar larvae are shifted to $29^{\circ} \mathrm{C}(n \geq 6){ }^{* * *} p \leq 0.001$. Error bars indicate SEM. 
conclusion stems from the finding that His2A-RFP, TUNEL-positive glia were heterogeneous in size, with many displaying very small nuclei (Fig. $7 B^{\prime}, B^{\prime \prime \prime}$, white arrows). This suggests that unlike most corpses, dying glial cells disappear. Moreover, unlike the majority of TUNELpositive cells, which were neurons, dying glial cells decreased in numbers with time (Fig. 7C), suggesting that they were cleared. These results suggest that the absence of $d r p r$ in glial cells can lead to autonomous cell death.

Uncleared apoptotic cells arise from a corpse processing defect in

drpr-deficient glia

$d r p r^{-1-}$ brains contain large numbers of persisting corpses as well as actively dying glia. The age-dependent neurodegeneration observed in these brains could be caused by either or both of these defects. To better understand the phenotype, we conducted a small screen to determine whether we could replicate the $d r p r$ phenotype. We knocked down other known interactors of $d r p r$, as well as other Drosophila engulfment genes. To our surprise, none of the candidates we tested, including shark, ced-6, and ced-12, displayed persistent apoptotic cells. However, Draper has been shown to be important for two distinct steps in phagocytosis: the recognition and the processing of the corpse (Kurant et al., 2008). The engulfment genes that we analyzed have been shown to be primarily involved in the recognition and/or cytoskeletal reorganization important for the internalization of the dead cell into the phagocyte (Doherty et al., 2009; Ziegenfuss et al., 2012). Therefore, we reasoned that the $d r p r^{-1-}$ phenotype may be due specifically to the corpse processing function of drpr.

To assess whether $d r p r$-deficient glia have defects in phagosome maturation, we coexpressed fluorescently tagged phagosome maturation markers in glial cells that also had drpr knocked down.

Failure of vesicles containing corpses to undergo fusion with organelles in the endocytic pathway has been shown to result in the accumulation of markers associated with corpse processing (Kinchen and Ravichandran, 2010). To this end, we expressed Rab7 and lysosomal-associated membrane protein 1 (LAMP)-GFP fusions, markers that are associated with corpse fusion to late endosome and lysosomes respectively ( $\mathrm{Lu}$ and Zhou, 2012). We found that when expressed alone, Rab7-GFP had a membraneous appearance and somewhat uniform distribution throughout the surface of the brain (Fig. $8 A^{\prime}, A^{\prime \prime}$ ). However, knocking down drpr in glia led to an accumulation of Rab7-GFP (Fig. $8 B^{\prime}, B^{\prime \prime}$ ). Specifically, Rab7-GFP accumu-
His2A-RFP
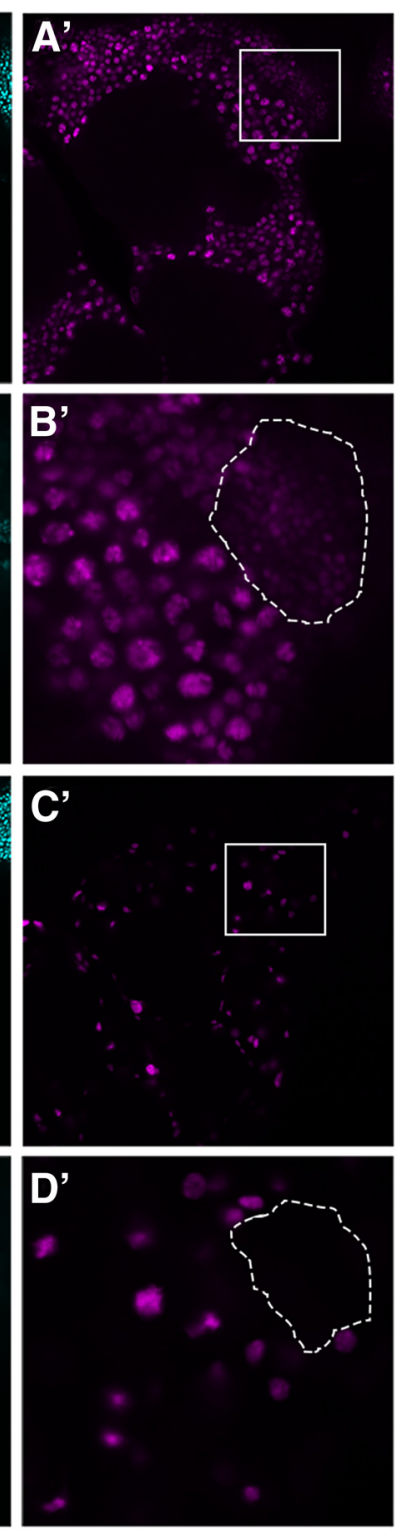
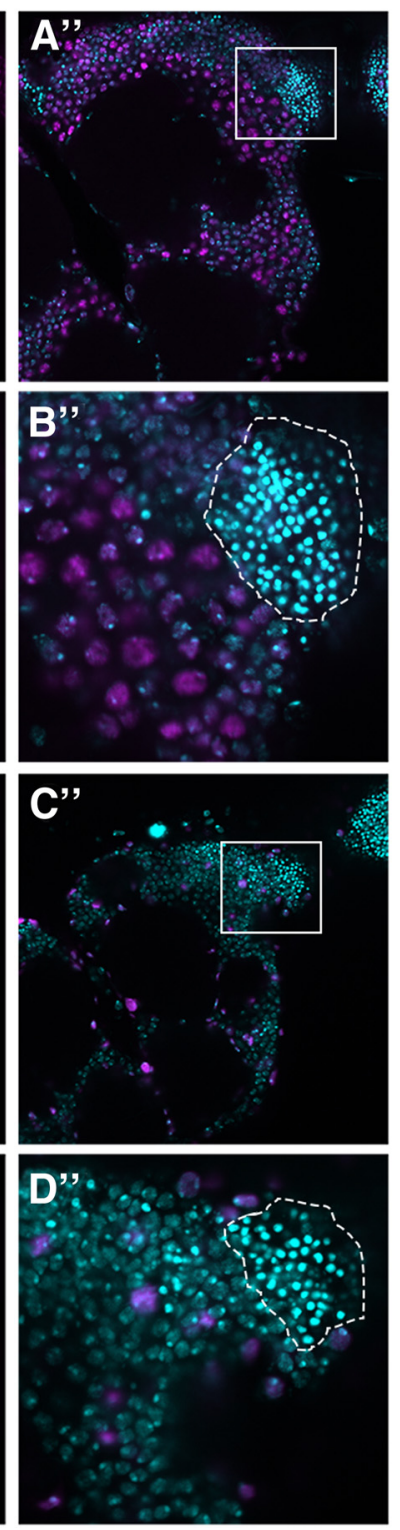

MERGE

Figure 5. Pyknotic nuclei largely correspond to neurons. $A-A^{\prime \prime}$, Pyknotic nuclei labeled with DAPI $(\boldsymbol{A})$ and His2A-RFP $\left(A^{\prime}\right)$ driven specifically in neurons with the elav-GAL4 promoter (elav-GAL4/+;UAS-His2A-RFP, $\mathrm{drpr}^{\Delta 5} / \mathrm{drpr}^{\Delta 5}$ ) colocalize with one another $\left(\boldsymbol{A}^{\prime \prime}\right) . \boldsymbol{B}-\boldsymbol{B}^{\prime \prime}$, Enlargements of white boxes in $\boldsymbol{A}-\boldsymbol{A}^{\prime \prime}$. Pyknotic nuclei in the outlined area $(\boldsymbol{B})$ colocalize with His2A-RFP in the same outlined area $\left(\boldsymbol{B}^{\prime}, \boldsymbol{B}^{\prime \prime}\right)$, although His-RFP is less intense than in some surrounding areas. $\left(-\boldsymbol{C}^{\prime \prime}\right.$, Pyknotic nuclei $(\boldsymbol{C})$ do not colocalize Enlargements of white boxes in $\boldsymbol{C}-\boldsymbol{C}^{\prime \prime}$. The outlined area with pyknotic nuclei $(\boldsymbol{D})$ is devoid of His2A-RFP $\left(\boldsymbol{D}^{\prime}, \boldsymbol{D}^{\prime \prime}\right)$, indicating that the majority of corpses are not glia.

lated and surrounded corpses but not healthy cells in these brains (Fig. $8 C^{\prime}-C^{\prime \prime \prime}$ ). LAMP-GFP had similar dynamics, where it appeared membrane-like and uniform in control brains (Fig. $8 D^{\prime}, D^{\prime \prime}$ ), but appeared to accumulate around corpses in brains where $d r p r$ RNAi was expressed in glia (Fig. $\left.8 E^{\prime}, E^{\prime \prime}\right)$. As with Rab7-GFP, LAMP-GFP surrounded corpses but not healthy cells in these brains (Fig. $\left.8 F^{\prime}-F^{\prime \prime \prime}\right)$. Unlike Rab7-GFP which seemed to "wrap" around corpses, LAMPGFP had a punctate appearance. The presence of both of these markers around corpses suggests that $d r p r$-deficient glia have defects in phagosome maturation. To further investigate whether corpses were internalized in draper mutants, we used 

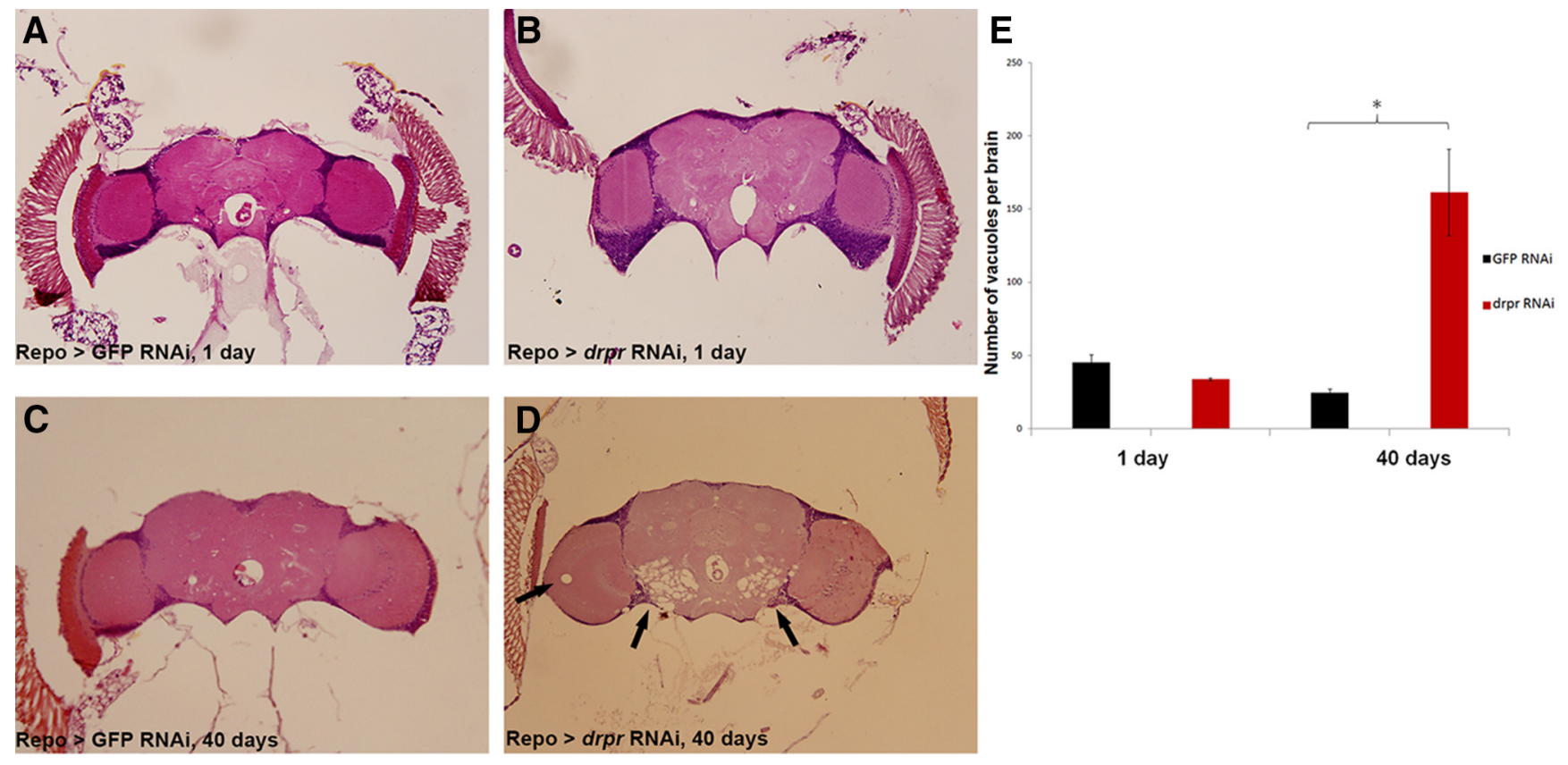

Figure 6. Knockdown of draper in glia leads to age-dependent neurodegeneration. $\boldsymbol{A}-\boldsymbol{D}$, Brain slices of UAS-GFP RNAi/+; repo-GAL4/+ (A) and UAS-draper-RNAi/+; repo-GAL4/+ (B) show little vacuolization at $1 \mathrm{~d}$ of age. However, at $40 \mathrm{~d}$ of age, draper RNAi brains (D) show significant levels of vacuolization (black arrows), whereas GFP RNAi brains (C) do not. $\boldsymbol{E}$, Quantification of the number of vacuoles per brains for GFP RNAi and draper RNAi brains at 1 and $40 \mathrm{~d}$, respectively $(n=6) .{ }^{*} p \leq 0.05$. Error bars indicate SEM.

DNA
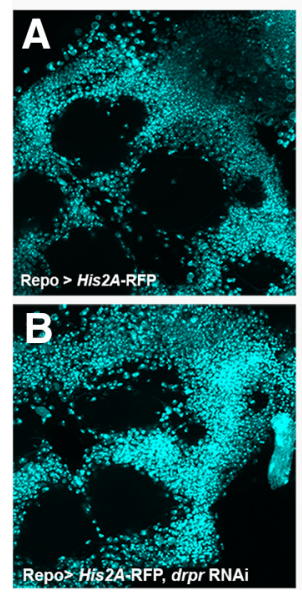

His2A-RFP
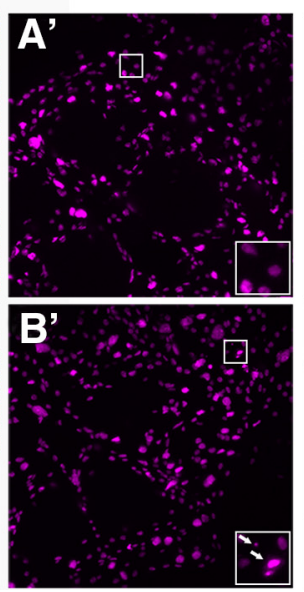

TUNEL
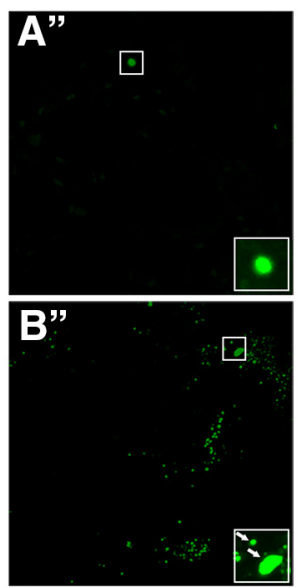

MERGE

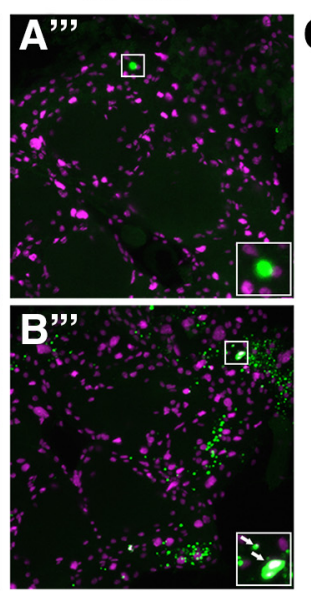

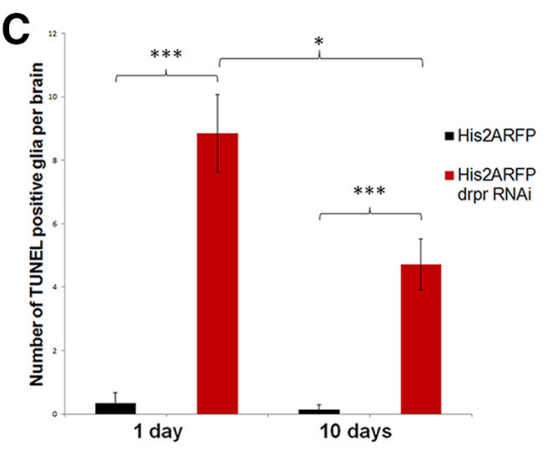

Figure 7. Draper-deficient glia actively undergo cell death. $\boldsymbol{A}-\boldsymbol{B}^{\prime \prime \prime}$, Glia-specific His2A-RFP does not colocalize with TUNEL in control brains (repo-GAL4/UAS-His2A-RFP; $\left.\boldsymbol{A}-\boldsymbol{A}^{\prime \prime \prime}\right)$, whereas His2A-RFP in glia expressing draper RNAi (UAS-draper-RNAi/+; repo-GAL4/UAS-His2A-RFP) shows some overlap with TUNEL staining (B-B'"). Insets represent enlargements of the white boxes. C, Quantification of the number of His2A-RFP positive cells that colabel with TUNEL for control and draper RNAi at 1 and $10 \mathrm{~d}$, respectively $(n \geq 6){ }^{*} p \leq 0.05$; ${ }^{* * *} p \leq$ 0.001. Error bars indicate SEM.

transmission electron microscopy. Under electron microscopy, wild-type cortex glia can be visualized easily because they stain more darkly than neurons (Fig. 9A-C). In control brains, we observed individual neuronal nuclei wrapped by cortex glia, but not internalized (Fig. 9C). Unlike control brains, we observed many corpses in $d r p r$ brains (Fig. $9 D-I$ ). In some cases, these corpses were surrounded by cytoplasm, suggesting they were inside glia (Fig. 9E,F). In other cases, corpses were clustered and surrounded by debris but it was not clear if they were within glia (Fig. 9H). In a few cases, corpses were isolated from glia, suggesting they had not been engulfed (Fig. 9I). These observations are consistent with our immu- nofluorescence studies indicating that $d r p r$-deficient glia have defects in corpse processing.

\section{Activating TORC1 in glial cells suppresses corpse accumulation and rescues neurodegeneration}

Our findings suggest that cellular corpses may result from defective phagosome processing in $d r p r$-deficient glia. It was shown previously that null mutants of transient receptor potential mucolipin (Trpml), a cation channel responsible for vesicle fusion, show high levels of late apoptotic cells and neurodegeneration (Venkatachalam et al., 2008). Defects in Trpml-dependent vesicle fusion can be rescued by TORC1 activation (Wong et al., 2012). 
Furthermore, it has been shown that gliaspecific knockdown of raptor, a component of TORC1, leads to defective phagocytosis in a model of axonal injury (Doherty et al., 2014). Therefore, TORC1 may be involved in apoptotic corpse processing during phagocytosis. Since our data indicate that corpse persistence in $d r \mathrm{pr}^{-1-}$ flies is a result of flawed phagosome maturation, we explored whether activation of TORC1 could modulate the $d r p r$ phenotype. Thus, we overexpressed Rheb, an activator of TORC1, in glial cells that also expressed $d r p r$ RNAi. Compared to expressing $d r p r$ RNAi by itself, expressing both Rheb and $d r p r$ RNAi led to a large reduction in the number of corpses forming in the brain. Flies mutant for raptor were shown to have lower levels of Draper expression, suggesting that the function of TORC1 could be to upregulate Draper (Doherty et al., 2014). To circumvent this potential issue, we expressed Rheb in glial cells in a $d r p r$ null background. repo-GAL4, $d r p r^{-1-}$ brains, like $d r p r^{-/-}$alone, displayed a large accumulation of apoptotic cells on the surface of the brain (Fig. $10 A^{\prime}, A^{\prime \prime}, F$ ). However, overexpressing Rheb in glia of drpr null flies strongly suppressed the phenotype close to wild-type levels (Fig. $\left.10 B^{\prime}, B^{\prime \prime}, F\right)$.

TORC1 activates a variety of different pathways important for cell growth, motility, proliferation, and protein translation (Betz and Hall, 2013). Of particular interest, TORC1 has been shown to negatively regulate the initiation of autophagy. Many proteins important in phagosome maturation are shared between both the autophagic and the phagocytic pathways (Martinez et al., 2011; Li et al., 2012). We therefore hypothesized that one of the roles of Drpr may be to act through TORC1 to inhibit the initiation of autophagy and prevent proteins from localizing to autophagosomes when they are needed for phagocytosis. To test whether autophagy is dysregulated in the absence of Drpr, we knocked down $d r p r$ in glia of flies that expressed Atg8a-GFP under the control of its endogenous promoter. We found that knocking down $d r p r$ in glia led to an increase in Atg8a puncta compared to control (Fig. 10C- $D^{\prime \prime}$ ). Furthermore, we found that the increased Atg8a tended to cluster around corpses (Fig. 10D- $D^{\prime \prime}$ ), suggesting that autophagy is dysregulated in $d r p r$ deficient glia that have internalized dead cells. To further investigate whether drpr-mediated suppression of autophagy is important for proper phagocytosis, we knocked down Atg1, the initiator of au-
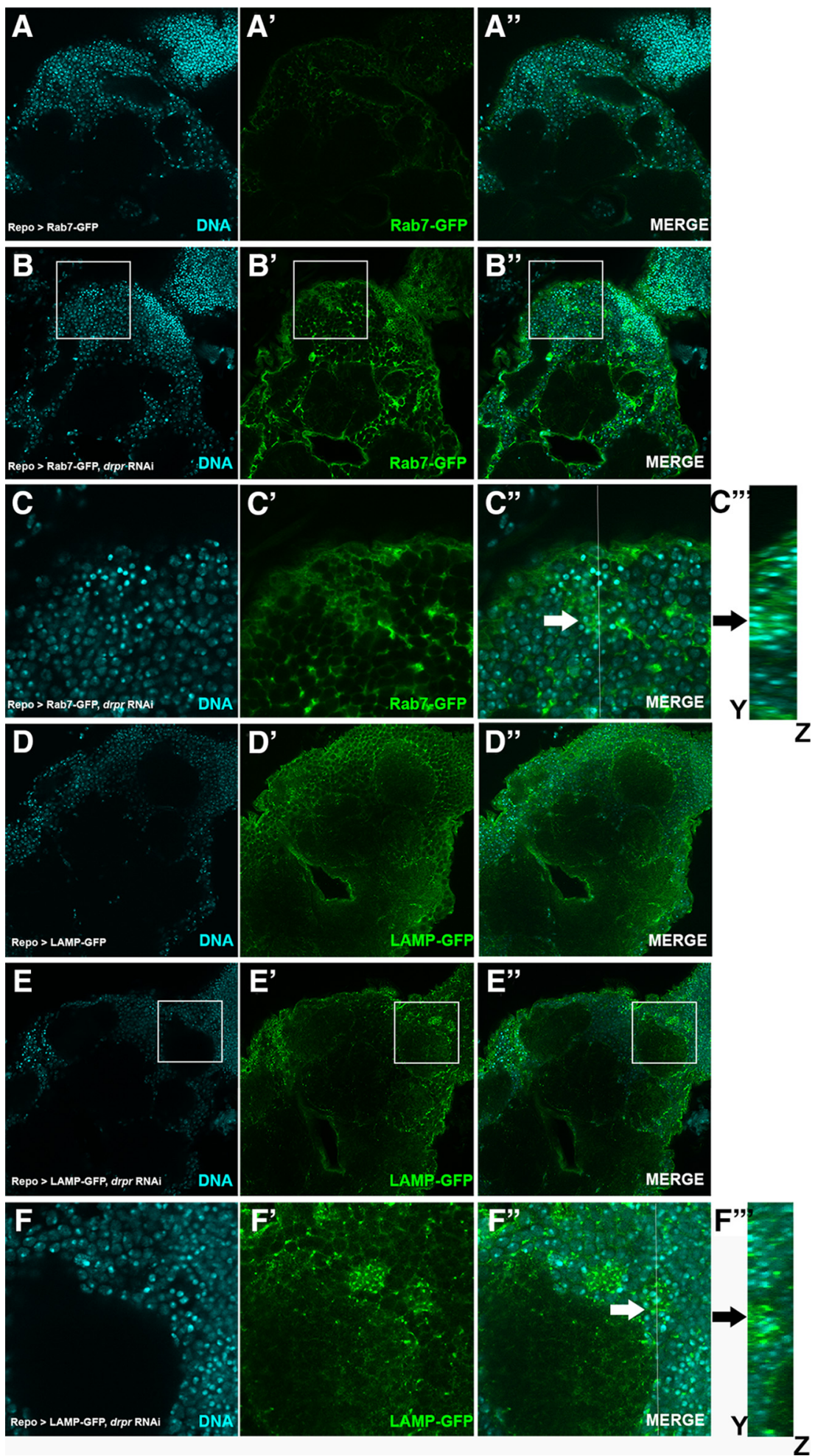

Figure 8. Corpses become arrested during late phagosome maturation. $\boldsymbol{A}-\boldsymbol{A}^{\prime \prime}$, Rab7-GFP driven in glia has a membrane like appearance in control (UAS-Rab7::GFP/+;repo-GAL4/+; $\left.\boldsymbol{A}^{\prime}, \boldsymbol{A}^{\prime \prime}\right)$. $\boldsymbol{B}-\boldsymbol{C}^{\prime \prime \prime}$, Rab7-GFP highly accumulates around pyknotic nuclei $\left(\boldsymbol{B}^{\prime}, \boldsymbol{B}^{\prime \prime}\right)$ in brains where draper has been knocked down in glia (UAS-Rab7::GFP/UAS-draper-RNAi; repo-GAL4/+). $\mathbf{C}-\boldsymbol{C}^{\prime \prime}$, Zoomed images of areas outlined by white rectangles in $\boldsymbol{B}-\boldsymbol{B}^{\prime \prime}$. $\boldsymbol{C}^{\prime \prime \prime}$ is a $Y-Z$ representation of the white line in $\boldsymbol{C}^{\prime}$. Rab7-GFP completely surrounds corpses but does not surround nuclei with normal nuclear morphology $\left(\boldsymbol{C}^{\prime \prime}, \boldsymbol{C}^{\prime \prime}\right)$. The white arrow indicates corpses in $\boldsymbol{C}^{\prime \prime}$ that are displayed in the $Y-Z$ projection in $C^{\prime \prime \prime}$ (black arrow). D-D', LAMP-GFP has a speckled as well as membranous appearance in control brains (UAS-LAMP::GFP/+; repo-GAL4/+) $\left(\boldsymbol{D}^{\prime}, \boldsymbol{D}^{\prime \prime}\right) . \boldsymbol{E}-\boldsymbol{E}^{\prime \prime}$, In brains where draper has been knocked down in glia (UAS-LAMP::GFP/UAS-draper-RNAi; repo-GAL4/+), LAMP-GFP speckles highly accumulate around pyknotic nuclei $\left(\boldsymbol{E}^{\prime}, \boldsymbol{E}^{\prime \prime}\right) . \boldsymbol{F}-\boldsymbol{F}^{\prime \prime}$, Enlargements of areas outlined by white rectangles in $\boldsymbol{E}_{-} \boldsymbol{E}^{\prime \prime} . \boldsymbol{F}^{\prime \prime \prime}$ is a $Y-Z$ representation of the white line in $\boldsymbol{E}^{\prime \prime}$. LAMP-GFP speckles completely surrounds corpses, but not nuclei with normal morphology $\left(\boldsymbol{F}^{\prime \prime}, \boldsymbol{F}^{\prime \prime \prime}\right)$. The white arrow indicates corpses in $\boldsymbol{F}^{\prime \prime}$ that are displayed in the $Y-Z$ projection in $\boldsymbol{F}^{\prime \prime \prime}$ (black arrow). 

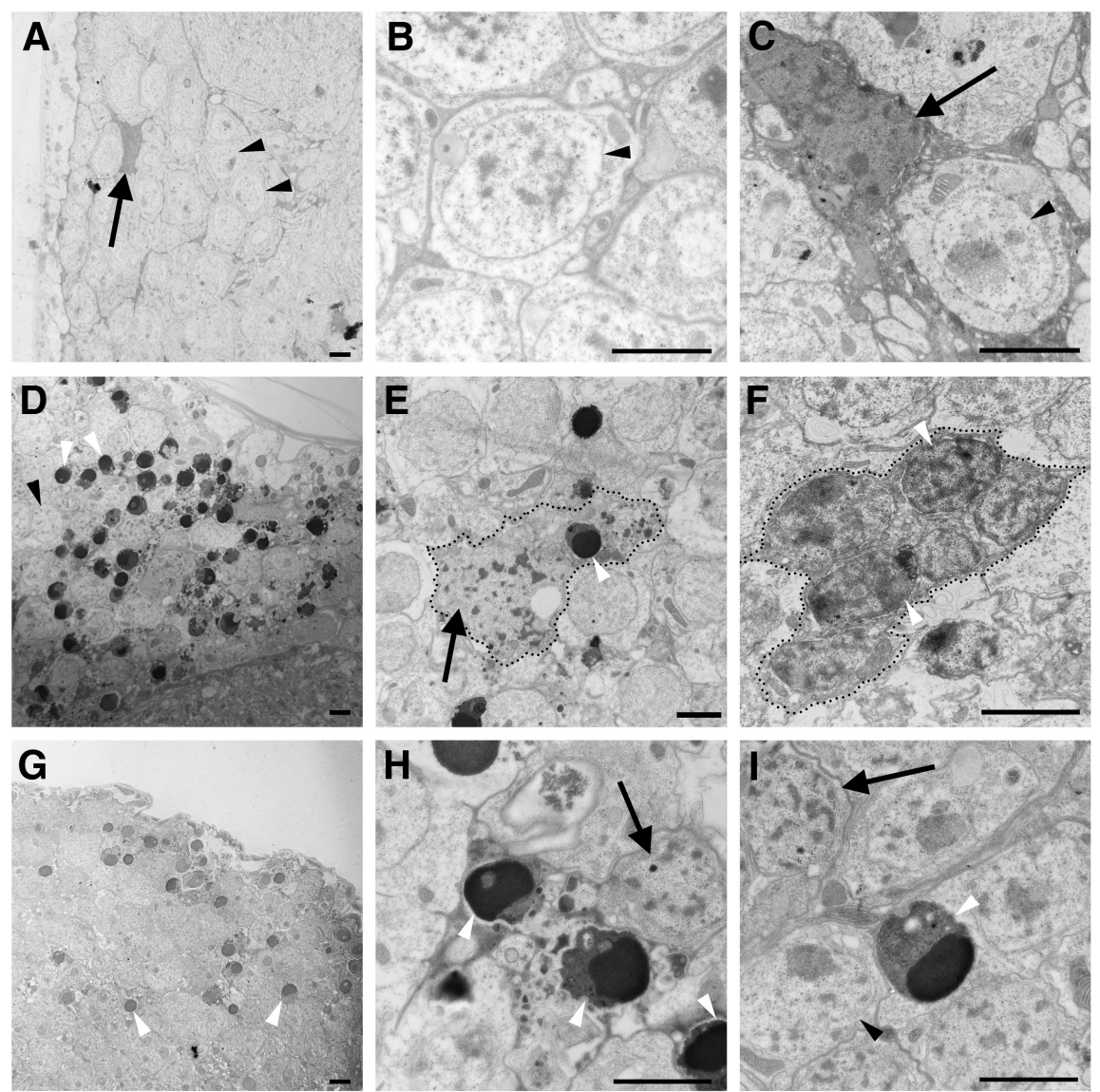

Figure 9. Electron microscopy shows that corpses accumulate and are associated with glia in drpr mutants. $\boldsymbol{A}-\boldsymbol{C}$, Images from control brains (repo-GAL4/+ or $w^{1118}$ ). A, An overview of control brain displays glia (gray cell, arrow) and neuronal cell bodies (lighter cells, black arrowheads). B, High-magnification image of neuronal cell bodies (black arrowhead) from a control brain. $\boldsymbol{C}$, High-magnification image of a glial cell (arrow) wrapping a neuron (black arrowhead). D-I, Images from drpr mutant brains. D, G, Overview of two different drpr brains show large numbers of corpses (white arrowheads) and healthy neuronal cell bodies (black arrowhead). $\boldsymbol{E}, \boldsymbol{F}$, High-magnification images from two different drpr brains show corpses (white arrowheads) inside glia (outlined). The arrow in $\boldsymbol{E}$ indicates a glial nucleus. $\boldsymbol{H}$, High-magnification image shows three corpses (white arrowheads) that are not separated by membranes and are adjacent to a glial nucleus (arrow). Visualization of the glial membrane is impeded by the amount of debris surrounding the corpses. I, An isolated corpse (white arrowhead) that does not appear to be within the neighboring glia (arrow). A normal cell body is indicated by a black arrowhead. Scale bars: $2 \mu \mathrm{m}$.

tophagy in Drosophila, in glia of $d r p r^{-1-}$ flies. We found that the accumulation of corpses in $\mathrm{drpr}^{-/-}$brains was strongly suppressed by knocking down Atg1 (Fig. $10 E, F$ ). We also found that knocking down Atg12 and Atg16 led to the suppression of corpse accumulation, but knockdowns of Atg7, Atg8, and Ref2P did not. These results suggest that TORC1 activation, through Atg1 inhibition, suppresses the corpse processing defect seen in $d \mathrm{rpr}^{-/-}$brains. Furthermore, TORC1 suppression of corpse accumulation persisted throughout the lifespan of the organism (Fig. 10F).

Two other phenotypes that we observed in $d r p r$ mutants were glial death and neurodegeneration. To determine whether glial death could be rescued by TORC1 activation, we overexpressed Rheb and His2A-RFP in glia in a $\mathrm{rrpr}^{-/-}$background. We found that the number of glia dying was similar in 1-d-old $d r \mathrm{pr}^{-1-}$ brains and $d r \mathrm{pr}^{-/-}$brains overexpressing Rheb in glia (Fig. $11 A-A^{\prime \prime \prime}, C$ ). These results were not due to Rheb's expression being dampened by coexpression of His2ARFP since the suppression of corpse accumulation was similar to expressing Rheb by itself (compare Figs. 10 $B^{\prime}, 11 A^{\prime \prime}$ ). These results suggest that the glia death is most likely due to a defect in the recognition role that Draper plays in phagocytosis, and not due to a corpse processing defect.

To determine whether the corpse processing role of $d r p r$ underlies agedependent neurodegeneration, we overexpressed Rheb in glia of $d r \mathrm{pr}^{-1-}$ flies and sectioned their brains. Forty-dayold $\mathrm{rrpr}^{-/-}$brains exhibited high levels of vacuolization (Fig. 12A,C). Fortyday-old $\mathrm{drpr}^{-/-}$brains overexpressing Rheb in glia, however, showed a decrease in the amount of vacuolization (Fig. $12 B, C$ ). This suggests that TORC1 activation in glia can suppress the agedependent neurodegeneration phenotype in $d r p r$ null brains. This finding, together with the finding that expressing Rheb in glia rescues corpse accumulation but not glial death, suggests that the persistence of corpses causes neurodegeneration. Therefore, defects in corpse processing, but not recognition, result in age-dependent neurodegeneration through the persistence of corpses in adult brains. These defects can be rescued by activating TORC1 in glia, which we show plays a novel role in phagosome maturation.

\section{Discussion}

How defects in the clearance of dead cells contribute to neurodegeneration has remained elusive. Our work has shown that the accumulation of apoptotic cells in the brain, due to defects in glia-mediated corpse processing, leads to neurodegeneration. We also showed that corpse accumulation as well as neurodegeneration can be suppressed by TORC1-mediated inhibition of autophagy. To our knowledge, it has not been shown previously that deficits in clearance of apoptotic cells by glia directly cause neurodegeneration in vivo.

Corpse accumulation in $\mathrm{drpr}^{-/-}$brains results from defects in phagosome maturation. This is evidenced by the accumulation of glia-derived Rab7-GFP and LAMP-GFP around neuronal pyknotic nuclei and the association of corpses with glia by electron microscopy. We showed that the defect in phagosome maturation can be rescued by activating TORC1. Activating TORC1 in glia of $\mathrm{drpr}^{-1-}$ flies suppressed corpse accumulation but not glia death. Since glia are still actively dying in these brains, it is unlikely that adult phagocytic defects associated with $\mathrm{drpr}^{-/-}$are suppressed through TORC1 activation. However, TORC1 activation suppressed age-dependent neurodegeneration. These findings indicate that processing defects in development, as opposed to phagocytic defects in adulthood, lead to persistent corpses. Our findings indicate that persistent corpses are responsible for age-dependent neurodegeneration in adulthood.

We found that corpses in adult $d r \mathrm{pr}^{-1-}$ brains formed during development. Corpse numbers remained constant even though the neurodegeneration phenotype indicated that 

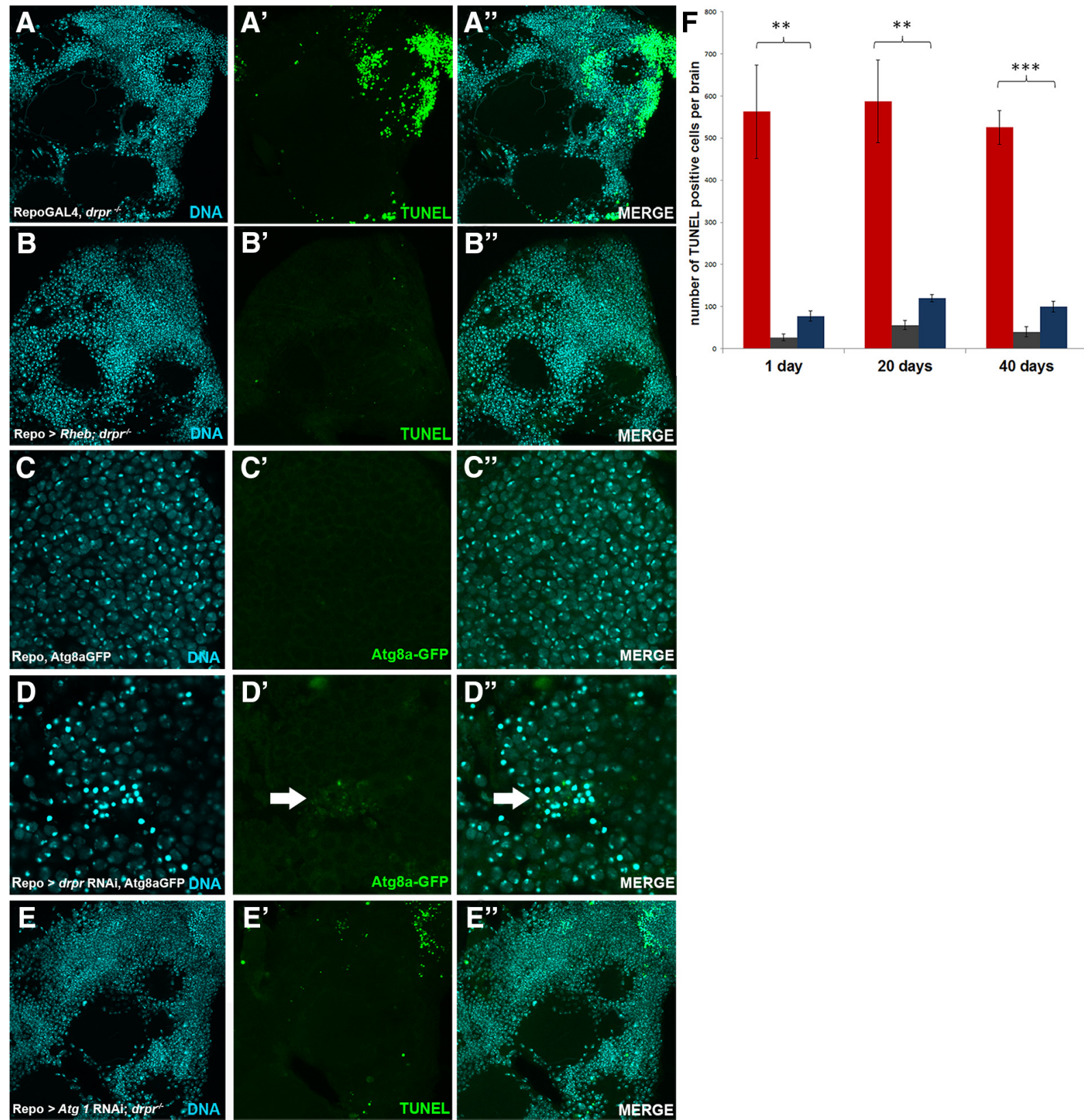

- RepoGAL4

drpr $-1-$

- Repo > Rheb

drpr $-/-$

- Repo > Atg 1

RNAi; drpr -/-

Figure 10. Activation of TORC1, through Atg1 inactivation, suppresses corpse accumulation and partially rescues neurodegeneration in draper null mutant brains. $A-B^{\prime \prime}$, The accumulation of TUNEL-positive cells $\left(\boldsymbol{A}^{\prime}, \boldsymbol{A}^{\prime \prime}\right)$ seen in draper null brains is suppressed by expressing Rheb specifically in glia (UAS-Rheb; drpr ${ }^{\Delta 5} /$ repo-GAL4, drpr $\left.{ }^{\Delta 5} ; \boldsymbol{B}^{\prime}, \boldsymbol{B}^{\prime \prime}\right)$. $\left(-\boldsymbol{D}^{\prime \prime}\right.$, Atg8a-GFP expression, driven by the endogenous promoter, is increased when drpr is knocked down in glia of $72 \mathrm{~h}$ pupae (UAS-draper-RNAi/+; repo-GAL4/Atg8aGFP; $\boldsymbol{D}^{\prime}$, white arrow) and clusters around corpses ( $\boldsymbol{D}^{\prime \prime}$, white arrow). Brains were stained with anti-GFP. $\boldsymbol{E}-\boldsymbol{E}^{\prime \prime}$, Knocking down Atg1 in glia of draper null flies (UAS-Atg1 RNAi; drpr ${ }^{\Delta 5} /$ repoGAL4, drpr $^{\Delta 5}$ ) phenocopies the TUNEL suppression $\left(\boldsymbol{E}^{\prime}, \boldsymbol{E}^{\prime \prime}\right)$ seen by overexpressing Rheb $\left(\boldsymbol{B}^{\prime}, \boldsymbol{B}^{\prime \prime}\right)$. $\boldsymbol{F}$, Quantification of TUNEL-positive corpses in draper null brains with repo-GAL4, repo-GAL4 UAS-Rheb, and UAS-Atg1 RNAi at 1, 20, and 40 d $(n \geq 5)$. ${ }^{* *} p \leq 0.01$; ${ }^{* * *} p \leq 0.001$. Error bars indicate SEM.

neurons were continuing to die in $d r p r^{-1-}$ brains. Why corpse accumulation does not increase in adults, even though neurodegeneration is occurring, is unclear. It may be because $d r p r$ has distinct roles at different stages of development. During embryogenesis, $d r p r$-deficient glia can still internalize dead cells, but are unable to process them (Kurant et al., 2008). Conversely, in adults, axotomy experiments showed that drpr-deficient glia fail to internalize debris, suggesting that after eclosion, Draper plays a stronger role in recognition (MacDonald et al., 2006). We propose that the critical role of Draper during larval development is in corpse processing.
Drosophila nervous system development is characterized by high levels of apoptosis. Thus, persistent corpses could arise from high levels of cell death overwhelming drpr-deficient glia. The increased intake of multiple apoptotic corpses would require efficient processing, and an inability to do so would lead to persistent corpses. The levels of cell death occurring in $d r p r^{-1-}$ brains during adulthood may be low enough to allow other recognition receptors and processing systems to ensure the removal of the corpses. This point could have relevance in situations of high cell death such as in disease or trauma. Specifically, during these conditions, there could be an increased need for very efficient processing. Individuals with 

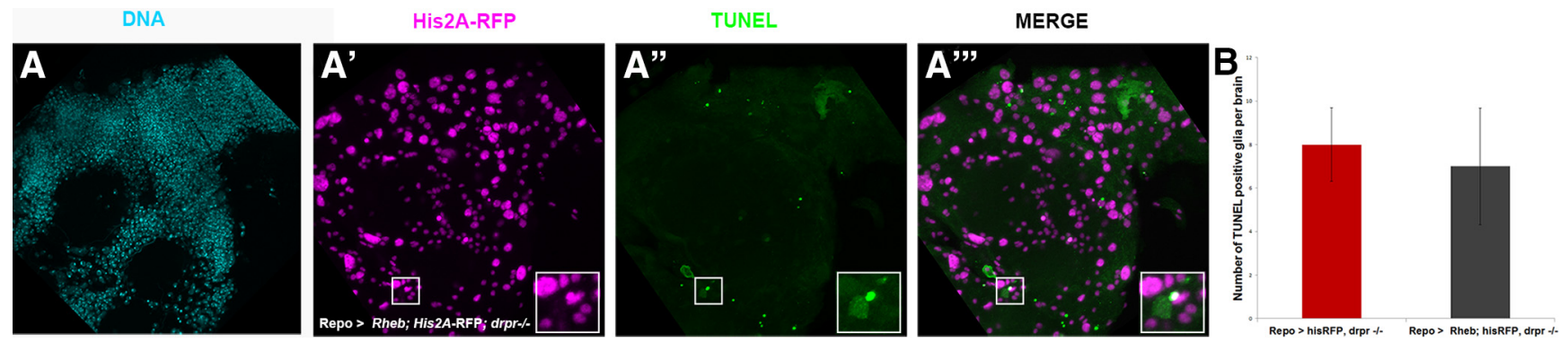

Figure 11. Activation of TORC1 does not rescue glia death in draper null flies. $A-A^{\prime \prime \prime}$, Glia-driven histone-RFP colocalizes with TUNEL staining in brains of draper null flies even when Rheb is overexpressed in glia (UAS-Rheb; UAS-His2A-RFP, drpr ${ }^{\Delta 5} / \mathrm{repo}_{\mathrm{GAL4}} \mathrm{drpr}^{\Delta 5}$ ). Insets represent enlargements of the white boxes. $\boldsymbol{B}$, Quantification of the number of histoneRFP-positive cells that colabel with TUNEL for drpr ${ }^{\Delta 5} /$ repoGAL4, $_{\text {drpr }}{ }^{\Delta 5}$ and UAS-Rheb/+;drpr ${ }^{\Delta 5} /$ repo-GAL4, $d r p r^{\Delta 5}$ at $1 \mathrm{~d}$ of age $(n \geq 4)$. Error bars indicate SEM.
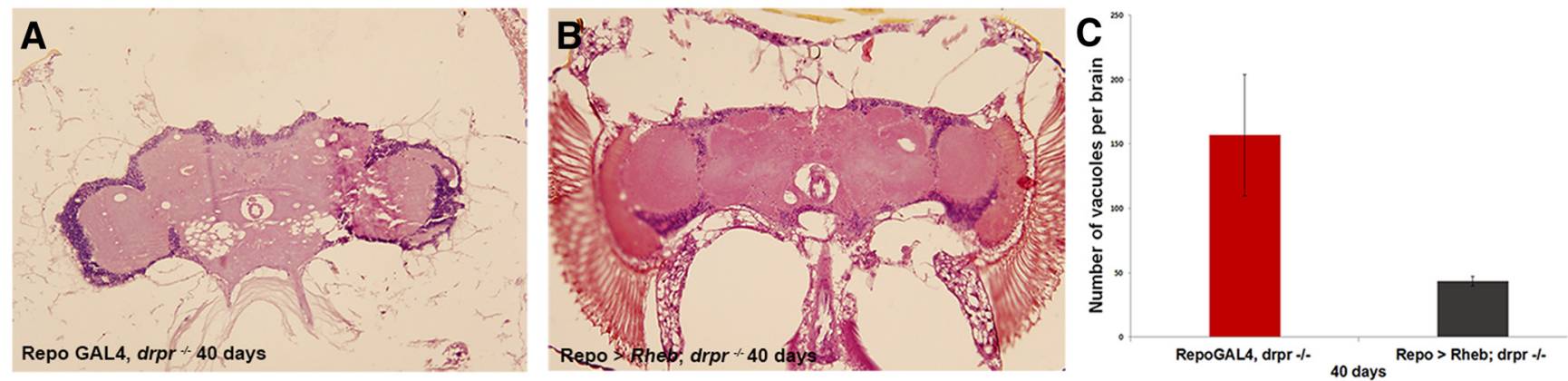

Figure 12. Activation of TORC 1 in glia rescues neurodegeneration seen in $\mathrm{drpr}^{-/-}$brains. $A, B$, Brain slices of $d r p r$ null flies expressing Rheb in glia show a decrease in the number of vacuoles at

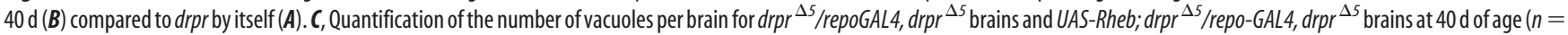
$6, p<0.06)$. Error bars indicate SEM.

defective alleles in genes for corpse processing, such as in TREM2, could be highly vulnerable to the persistence of corpses and further exacerbations of neurodegeneration.

How corpse processing defects cause neurodegeneration remains to be elucidated. It is unlikely that the corpses themselves cause death to the surrounding cells. This stems from the finding that the onset of neurodegeneration is age dependent even though the corpses are present from earlier in development. It is therefore more likely that the presence of corpses interferes with normal neuronal or glia development/ function and this then leads to neurodegeneration. Previous work has shown that phagocytosis is important in regulating the number of neural precursor cells in mammalian development as well as in adults (Lu et al., 2011; Cunningham et al., 2013). It may be that defective corpse processing in $d r p r^{-1-}$ brains results in aberrant neural stem cell production. Furthermore, in mice it was found that the MeCP2 mutation that causes Rett syndrome, an autism spectrum disorder, led to defective phagocytosis in glia (Derecki et al., 2012). Although not a neurodegenerative disease, patients with Rett syndrome do display increased cell loss in the cerebellum (Oldfors et al., 1990). Therefore, corpse processing defects observed in $\mathrm{drpr}^{-1-}$ could lead to developmental defects which then give rise to age dependent neurodegeneration.

Previous work has indirectly implicated TORC1 in phagocytosis. Raptor, a component of TORC1, as well as the phosphoinositide 3-kinase (PI3K) signaling pathway, an activator of TORC1, have been shown to be important for phagocytosis in Drosophila (Doherty et al., 2014). Furthermore, in both mammals and C. elegans, disrupting PI3K signaling leads to defects in the corpse processing of bacteria and apoptotic cells, respectively (Fratti et al., 2001; Kinchen et al., 2008). Our work, in conjunction with these previous findings, suggests that TORC1 acts downstream of PI3K to regulate phagosome maturation.

We found that the accumulation of corpses was rescued by knocking down Atg1 in glia. This suggests that TORC1 promotes corpse processing by inhibiting autophagy initiation. At first glance, this seems contrary to data accumulated both in C. elegans and mammals. Studies have shown that autophagy proteins localize to vesicles containing corpses, allowing for proper processing of dead cells, in a process known as LC3-associated phagocytosis (LAP; Florey et al., 2011; Martinez et al., 2011; Li et al., 2012). LAP, however, does not appear to require Atg1 or the autophagy initiation process, but does require Atg8 (the Drosophila homolog of LC3) (Florey et al., 2011; Martinez et al., 2011; Li et al., 2012). This is consistent with our findings that knocking down Atg1 but not Atg8 leads to the suppression of corpse accumulation. Moreover, we do see Atg-GFP puncta in the vicinity of corpses in draper mutants.

Phagocytosis has been shown to be an energy-intensive process (Han and Ravichandran, 2011). Autophagy initiation may need to be suppressed to prevent the formation of autophagosomes and autolysosomes (which form under conditions of high energy demand). This would then free up other autophagy proteins and allow their recruitment to vesicles containing corpses. Within the endocytosis pathway, some proteins, such as Rab 5 and Rab7, have been shown to be important for many membrane trafficking events. TORC1mediated inhibition of autophagy may also be important in preventing these proteins from localizing to autophagosomes. This would allow their proper localization to vesicles containing corpses. Autophagy and phagocytosis are essential processes in most multicellular organisms. Autophagy inhibition 
may have evolved across species as a key mechanism to promote the proper localization of trafficking proteins during phagocytosis.

\section{References}

Awasaki T, Lai SL, Ito K, Lee T (2008) Organization and postembryonic development of glial cells in the adult central brain of Drosophila. J Neurosci 28:13742-13753. CrossRef Medline

Betz C, Hall MN (2013) Where is mTOR and what is it doing there? J Cell Biol 203:563-574. CrossRef Medline

Blander JM, Medzhitov R (2006) On regulation of phagosome maturation and antigen presentation. Nat Immunol 7:1029-1035. CrossRef Medline

Brand AH, Perrimon N (1993) Targeted gene-expression as a means of altering cell fates and generating dominant phenotypes. Development 118: 401-415. Medline

Cantoni C, Bollman B, Licastro D, Xie M, Mikesell R, Schmidt R, Yuede CM, Galimberti D, Olivecrona G, Klein RS, Cross AH, Otero K, Piccio L (2015) TREM2 regulates microglial cell activation in response to demyelination in vivo. Acta Neuropathol 129:429-447. CrossRef Medline

Celotto AM, Palladino MJ (2005) Drosophila: a "model" model system to study neurodegeneration. Mol Interventions 5:292-303. CrossRef

Chung WS, Clarke LE, Wang GX, Stafford BK, Sher A, Chakraborty C, Joung J, Foo LC, Thompson A, Chen C, Smith SJ, Barres BA (2013) Astrocytes mediate synapse elimination through MEGF10 and MERTK pathways. Nature 504:394-400. CrossRef Medline

Cunningham CL, Martínez-Cerdeño V, Noctor SC (2013) Microglia regulate the number of neural precursor cells in the developing cerebral cortex. J Neurosci 33:4216-4233. CrossRef Medline

Derecki NC, Cronk JC, Lu Z, Xu E, Abbott SB, Guyenet PG, Kipnis J (2012) Wild-type microglia arrest pathology in a mouse model of Rett syndrome. Nature 484:105-105. CrossRef Medline

Doherty J, Logan MA, Tasdemir OE, Freeman MR (2009) Ensheathing glia function as phagocytes in the adult Drosophila brain. J Neurosci 29: 4768-4781. CrossRef Medline

Doherty J, Sheehan AE, Bradshaw R, Fox AN, Lu TY, Freeman MR (2014) PI3K signaling and Stat92E converge to modulate glial responsiveness to axonal injury. PLoS Biol 12:e1001985. Medline

Draper I, Mahoney LJ, Mitsuhashi S, Pacak CA, Salomon RN, Kang PB (2014) Silencing of drpr leads to muscle and brain degeneration in adult Drosophila. Am J Pathol 184:2653-2661. CrossRef Medline

Edwards TN, Meinertzhagen IA (2010) The functional organisation of glia in the adult brain of Drosophila and other insects. Prog Neurobiol 90: 471-497. CrossRef Medline

Elliott MR, Ravichandran KS (2010) Clearance of apoptotic cells: implications in health and disease. J Cell Biol 189:1059-1070. CrossRef Medline

Etchegaray JI, Timmons AK, Klein AP, Pritchett TL, Welch E, Meehan TL, Li C, McCall K (2012) Draper acts through the JNK pathway to control synchronous engulfment of dying germline cells by follicular epithelial cells. Development 139:4029-4039. CrossRef Medline

Florey O, Kim SE, Sandoval CP, Haynes CM, Overholtzer M (2011) Autophagy machinery mediates macroendocytic processing and entotic cell death by targeting single membranes. Nat Cell Biol 13:1335-1118. Medline

Fratti RA, Backer JM, Gruenberg J, Corvera S, Deretic V (2001) Role of phosphatidylinositol 3-kinase and Rab5 effectors in phagosomal biogenesis and mycobacterial phagosome maturation arrest. J Cell Biol 154: 631-644. CrossRef Medline

Freeman MR, Delrow J, Kim J, Johnson E, Doe CQ (2003) Unwrapping glial biology: $\mathrm{Gcm}$ target genes regulating glial development, diversification, and function. Neuron 38:567-580. CrossRef Medline

Frost B, Hemberg M, Lewis J, Feany MB (2014) Tau promotes neurodegeneration through global chromatin relaxation. Nat Neurosci 17:357-366. CrossRef Medline

Fu R, Shen Q, Xu P, Luo JJ, Tang Y (2014) Phagocytosis of microglia in the central nervous system diseases. Mol Neurobiol 49:1422-1434. CrossRef Medline

Fullard JF, Kale A, Baker NE (2009) Clearance of apoptotic corpses. Apoptosis 14:1029-1037. CrossRef Medline

Gilbert LI (2012) Insect molecular biology and biochemistry, Ed 1. Waltham, MA: Elsevier/Academic.

Guerreiro R, Wojtas A, Bras J, Carrasquillo M, Rogaeva E, Majounie E, Cruchaga C, Sassi C, Kauwe JS, Younkin S, Hazrati L, Collinge J, Pocock J, Lashley T, Williams J, Lambert JC, Amouyel P, Goate A, Rademakers R,
Morgan K, et al. (2013) TREM2 variants in Alzheimer's disease. N Engl J Med 368:117-127. CrossRef Medline

Han CZ, Ravichandran KS (2011) Metabolic connections during apoptotic cell engulfment. Cell 147:1442-1445. CrossRef Medline

Hegde VR, Vogel R, Feany MB (2014) Glia are critical for the neuropathology of complex I deficiency in Drosophila. Hum Mol Genet 23:46864692. CrossRef Medline

Hochreiter-Hufford A, Ravichandran KS (2013) Clearing the dead: apoptotic cell sensing, recognition, engulfment, and digestion. Cold Spring Harbor Perspect Biol 5:a008748. CrossRef

Ito K, Shinomiya K, Ito M, Armstrong JD, Boyan G, Hartenstein V, Harzsch S, Heisenberg M, Homberg U, Jenett A, Keshishian H, Restifo LL, Rössler W, Simpson JH, Strausfeld NJ, Strauss R, Vosshall LB, Grp IBNW (2014) A systematic nomenclature for the Insect Brain. Neuron 81:755-765. CrossRef Medline

Jonsson T, Stefansson H, Steinberg S, Jonsdottir I, Jonsson PV, Snaedal J, Bjornsson S, Huttenlocher J, Levey AI, Lah JJ, Rujescu D, Hampel H, Giegling I, Andreassen OA, Engedal K, Ulstein I, Djurovic S, IbrahimVerbaas C, Hofman A, Ikram MA, et al. (2013) Variant of TREM2 associated with the risk of Alzheimer's disease. N Engl J Med 368:107-116. CrossRef Medline

Kauwe JS, Bailey MH, Ridge PG, Perry R, Wadsworth ME, Hoyt KL, Staley LA, Karch CM, Harari O, Cruchaga C, Ainscough BJ, Bales K, Pickering EH, Bertelsen S, Fagan AM, Holtzman DM, Morris JC, Goate AM, Neuroimaging AD (2014) Genome-wide association study of CSF levels of 59 Alzheimer's disease candidate proteins: significant associations with proteins involved in amyloid processing and inflammation. Plos Genet 10:e1004758. CrossRef.

Kinchen JM, Ravichandran KS (2010) Identification of two evolutionarily conserved genes regulating processing of engulfed apoptotic cells. Nature 464:778-782. CrossRef Medline

Kinchen JM, Doukoumetzidis K, Almendinger J, Stergiou L, ToselloTrampont A, Sifri CD, Hengartner MO, Ravichandran KS (2008) A pathway for phagosome maturation during engulfment of apoptotic cells. Nat Cell Biol 10:556-566. CrossRef Medline

Kurant E, Axelrod S, Leaman D, Gaul U (2008) Six-microns-under acts upstream of draper in the glial phagocytosis of apoptotic neurons. Cell 133:498 -509. CrossRef Medline

Li W, Zou W, Yang Y, Chai Y, Chen B, Cheng S, Tian D, Wang X, Vale RD, Ou G (2012) Autophagy genes function sequentially to promote apoptotic cell corpse degradation in the engulfing cell. J Cell Biol 197:27-35. CrossRef Medline

Logan MA, Hackett R, Doherty J, Sheehan A, Speese SD, Freeman MR (2012) Negative regulation of glial engulfment activity by Draper terminates glial responses to axon injury. Nat Neurosci 15:722-730. CrossRef Medline

Lu N, Zhou Z (2012) Membrane trafficking and phagosome maturation during the clearance of apoptotic cells. Int Rev Cel Mol Biol 293:269-309. CrossRef

Lu Z, Elliott MR, Chen Y, Walsh JT, Klibanov AL, Ravichandran KS, Kipnis J (2011) Phagocytic activity of neuronal progenitors regulates adult neurogenesis. Nat Cell Biol 13:1076-1083. CrossRef Medline

MacDonald JM, Beach MG, Porpiglia E, Sheehan AE, Watts RJ, Freeman MR (2006) The Drosophila cell corpse engulfment receptor draper mediates glial clearance of severed axons. Neuron 50:869-881. CrossRef Medline

Martinez J, Almendinger J, Oberst A, Ness R, Dillon CP, Fitzgerald P, Hengartner MO, Green DR (2011) Microtubule-associated protein 1 light chain 3 alpha (LC3)-associated phagocytosis is required for the efficient clearance of dead cells. Proc Natl Acad Sci U S A 108:17396-17401. CrossRef Medline

McGuire SE, Le PT, Osborn AJ, Matsumoto K, Davis RL (2003) Spatiotemporal rescue of memory dysfunction in Drosophila. Science 302: 1765-1768. CrossRef Medline

McPhee CK, Logan MA, Freeman MR, Baehrecke EH (2010) Activation of autophagy during cell death requires the engulfment receptor Draper. Nature 465:1093-1096. Medline

Oldfors A, Sourander P, Armstrong DL, Percy AK, Witt-Engerström I, Hagberg BA (1990) Rett syndrome-Cerebellar Pathology. Pediatr Neurol 6:310-314. CrossRef Medline

Poon IK, Lucas CD, Rossi AG, Ravichandran KS (2014) Apoptotic cell clearance: basic biology and therapeutic potential. Nat Rev Immunol 14: 166-180. CrossRef Medline

Savill J, Dransfield I, Gregory C, Haslett C (2002) A blast from the past: 
clearance of apoptotic cells regulates immune responses. Nat Rev Immunol 2:965-975. CrossRef Medline

Scheib JL, Sullivan CS, Carter BD (2012) Jedi-1 and MEGF10 signal engulfment of apoptotic neurons through the tyrosine kinase Syk. J Neurosci 32:13022-13031. CrossRef Medline

Shulman JM, Shulman LM, Weiner WJ, Feany MB (2003) From fruit fly to bedside: translating lessons from Drosophila models of neurodegenerative disease. Curr Opin Neurol 16:443-449. Medline

Sierra A, Abiega O, Shahraz A, Neumann H (2013) Janus-faced microglia: beneficial and detrimental consequences of microglial phagocytosis. Front Cell Neurosci 7:6. Medline

Song Z, McCall K, Steller H (1997) DCP-1, a Drosophila cell death protease essential for development. Science 275:536-540. CrossRef Medline

Venkatachalam K, Long AA, Elsaesser R, Nikolaeva D, Broadie K, Montell C (2008) Motor deficit in a Drosophila model of mucolipidosis type
IV due to defective clearance of apoptotic cells. Cell 135:838-851. CrossRef Medline

Wong CO, Li R, Montell C, Venkatachalam K (2012) Drosophila TRPML is required for TORC1 activation. Curr Biol 22:1616-1621. CrossRef Medline

Yu XM, Lu N, Zhou Z (2008) Phagocytic receptor CED-1 initiates a signaling pathway for degrading engulfed apoptotic cells. PLoS Biol 6:e61. Medline

Yvan-Charvet L, Pagler TA, Seimon TA, Thorp E, Welch CL, Witztum JL, Tabas I, Tall AR (2010) ABCA1 and ABCG1 protect against oxidative stress-induced macrophage apoptosis during efferocytosis. Circ Res 106: 1861-1869. CrossRef

Ziegenfuss JS, Doherty J, Freeman MR (2012) Distinct molecular pathways mediate glial activation and engulfment of axonal debris after axotomy. Nat Neurosci 15:979-987. CrossRef Medline 